\title{
Analytical soft SUSY spectrum in mirage-type mediation scenarios
}

\author{
Fei Wang \\ Department of Physics and Engineering, Zhengzhou University, \\ Zhengzhou 450000, P.R. China \\ State Key Laboratory of Theoretical Physics, Institute of Theoretical Physics, \\ Chinese Academy of Sciences, \\ Beijing 100190, P.R. China \\ E-mail: feiwang@zzu.edu.cn
}

ABSTRACT: We derive explicitly the soft SUSY breaking parameters at arbitrary low energy scale in the (deflected) mirage type mediation scenarios with possible gauge or Yukawa mediation contributions. Based on the Wilsonian effective action after integrating out the messengers, we obtain analytically the boundary value (at the GUT scale) dependencies of the effective wavefunctions and gauge kinetic terms. Note that the messenger scale dependencies of the effective wavefunctions and gauge kinetic terms had already been discussed in GMSB. The RGE boundary value dependencies, which is a special feature in (deflected) mirage type mediation, is the key new ingredients in this study. The appearance of 'mirage' unification scale in mirage mediation is proved rigorously with our analytical results. We also discuss briefly the new features in deflected mirage mediation scenario in the case the deflection comes purely from the Kahler potential and the case with messengermatter interactions.

Keywords: Supersymmetry Breaking, Supersymmetric Effective Theories, Compactification and String Models, Supersymmetric Standard Model

ARXIV EPRINT: 1808.08529 


\section{Contents}

1 Introduction 1

2 Brief review of the mirage type mediation scenarios 3

3 Analytical expressions of soft SUSY breaking parameters 4

3.1 Gaugino mass 5

3.2 Trilinear terms 6

$\begin{array}{lll}3.3 & \text { Soft scalar masses } & 12\end{array}$

4 Applications of the general analytical results $\quad 13$

$\begin{array}{lll}4.1 & \text { Analytical results for mirage mediation } & 13\end{array}$

4.2 Deflection in mirage mediation from the Kahler potential 14

$\begin{array}{lll}4.3 & \text { Deflected mirage mediation with messenger-matter interactions } & 15\end{array}$

$\begin{array}{lll}5 & \text { Conclusions } & 17\end{array}$

$\begin{array}{ll}\text { A Coefficients in wavefunction expansion } & 17\end{array}$

$\begin{array}{lr}\text { B Low energy spectrum in deflected mirage mediation } & 18\end{array}$

\section{Introduction}

After the discovery of the $125 \mathrm{GeV}$ Higgs boson in 2012 at the CERN LHC [1, 2], the long missing particle content of the Standard Model(SM) has finally been verified. In spite of the impressive triumph of SM, many physicists still believe that new physics may be revealed at LHC. Among the many new physics models that can solve the fine-tuning problem, the most elegant and compelling resolution is low energy supersymmetry. Augmented with weak scale soft SUSY breaking terms, the quadratic cutoff dependence is absent, leaving only relatively mild but intertwined logarthmic sensitivity to high scale physics. As such soft SUSY breaking spectrum is determined by the SUSY breaking mechanism, it is interesting to survey the phenomenology related to supersymmetry breaking mechanism.

In Type IIB string theory compactified on a Calabi-Yau (CY) orientifold, the presence of NS and RR 3-form background fluxes can fix the dilaton and the complex structure moduli, leaving only the Kahler moduli in the Wilsonian effective supergravity action after integrating out the superheavy complex structure moduli and dilaton. The remaining Kahler moduli fields could be stabilized by non-perturbative effects, such as instanton or gaugino condensation. In order to generates SUSY breaking in the observable sector and obtain a very tiny positive cosmological constant, Kachru-Kallosh-Linde- Trivedi (KKLT) [3] propose to add an anti-D3 brane at the tip of the Klebanov-Strassler throat (or adding F-term, D-term SUSY breaking contributions [4-6]) to explicitly break SUSY 
and lift the AdS universe to obtain a dS one. In addition to the anomaly mediation contributions, SUSY breaking effects from the light Kahler moduli fields could also be mediated to the visible sector and result in a mixed modulus-anomaly mediation SUSY breaking scenario $[7,8]$. It is interesting to note that the involved modulus mediated SUSY breaking contributions can be comparable to that of the anomaly mediation $[9,10]$. With certain assumptions on the Yukawa couplings and the modular weights, the SUSY breaking contributions from the renormalization group running and anomaly mediation could cancel each other at a 'mirage' unification scale, leading to a compressed low energy SUSY breaking spectrum [11]. Such a mixed modulus-anomaly mediation SUSY breaking mechanism is dubbed as 'mirage mediation'.

Anomaly mediation contribution is a crucial ingredient of such a mixed modulusanomaly mediation. It is well known that the pure anomaly mediation is bothered by the tachyonic slepton problem [12-18]. One of its non-trivial extensions with messenger sectors, namely the deflected anomaly mediated SUSY breaking (AMSB), can elegantly solve such a tachyonic slepton problem through the deflection of the renormalization group equation (RGE) trajectory [19-25]. Such a messenger sector can also be present in the mirage mediation so that additional gauge contributions by the messengers [26-28] can deflect the RGE trajectory and change the low energy soft SUSY predictions. Additional deflection in mirage mediation can be advantageous in phenomenological aspect. For example, apparent gaugino mass unification at $\mathrm{TeV}$ scale could still be realized with the simplest 'no scale' Kahler potential, which, in ordinary mirage mediation, can only be possible with the not UV-preferable $\alpha=2$ case. Relevant discussions on mirage-type mediation scenarios can be seen, for example, in [29-39].

In mirage type mediation scenarios, analytical expressions for the soft SUSY breaking parameters are no not given at the messenger scale $M$ (or scale below $M$ ), but given at the GUT scale instead. One needs to numerically evolve the spectrum with GUT scale input to obtain the low energy SUSY spectrum. This procedure obscures the appearance of 'mirage' unification scale from the input. In mirage mediation scenarios with deflection from Kahler potential, analytical results of mirage mediation are necessary to predict the low energy SUSY spectrum. So it is preferable to give the analytical expressions for the soft SUSY breaking parameters in mirage type mediation scenarios at arbitrary low energy scale. Besides, possible new Yukawa-type interactions involving the messengers may give additional Yukawa mediation contributions to the low energy soft SUSY spectrum (See [40] for example). Such a generalization of deflected mirage mediation scenario shows new features in phenomenological studies. The inclusion of Yukawa mediation contributions at (or below) the messenger scale $M$ are non-trivial and again prefer analytical expressions near the messenger scale.

This paper is organized as follows. We briefly review the mirage type mediation scenarios in section 2. A general discussion on the analytical expressions for the soft SUSY parameters in the generalized deflected mirage mediation is given in section 3 . We discuss some applications of our analytical results in section 4, including the proof of the 'mirage' unification scale in mirage mediation with our analytical results and the discussions on deflection from Kahler potential. Section 5 contains our conclusions. 


\section{Brief review of the mirage type mediation scenarios}

Inspired by string-motivated KKLT approach to moduli stabilization within Type IIB string theory, mirage mediation supersymmetry breaking is proposed, in which the modulus mediated supersymmetry breaking terms are suppressed by numerically a loop factor so that the anomaly mediated terms can be competitive.

After fixing and integrating out the dilaton and the complex structure moduli, the four-dimensional Wilsonian effective supergravity action (defined at the boundary scale $\Lambda$ ) in terms of compensator field and a single Kahler modulus parameterizing the overall size of the compact space [11] is given as

$$
e^{-1} \mathcal{L}=\int d^{4} \theta\left[\phi^{\dagger} \phi\left(-3 e^{-K / 3}\right)-\left(\phi^{\dagger} \phi\right)^{2} \bar{\theta}^{2} \theta^{2} \mathcal{P}_{\text {lift }}\right]+\int d^{2} \theta \phi^{3} W+\int d^{2} \theta \frac{f_{i}}{4} W_{i}^{a} W_{i}^{a}
$$

with a holomorphic gauge kinetic term

$$
f_{i}=\frac{1}{g_{i}^{2}}+i \frac{\theta}{8 \pi}
$$

The Kahler potential takes the form

$$
\begin{aligned}
K= & -3 \ln \left(T+T^{\dagger}\right)+Z_{X}\left(T^{\dagger}, T\right) X^{\dagger} X+Z_{\Phi}\left(T^{\dagger}, T\right) \Phi^{\dagger} \Phi \\
& +\sum_{i} Z_{P_{i}, \bar{P}_{i}}\left(T^{\dagger}, T\right)\left[P_{i}^{\dagger} P_{i}+\bar{P}_{i}^{\dagger} \bar{P}_{i}\right],
\end{aligned}
$$

with the 'no-scale' kinetic term for the Kahler modulus $T$. The gauge kinetic term $f_{i}$, the messenger superfields $P_{i}$, the MSSM superfields $\Phi$ and the pseudo-moduli superfields are all assumed to depend non-trivially on the Kahler moduli $T$ as

$$
\begin{aligned}
Z_{X}\left(T^{\dagger}, T\right) & =\frac{1}{\left(T^{\dagger}+T\right)^{n_{X}}}, & Z_{\Phi}\left(T^{\dagger}, T\right) & =\frac{1}{\left(T^{\dagger}+T\right)^{n_{\Phi}}}, \\
f_{i}(T) & =T^{l_{i}}, & Z_{P_{i}, \bar{P}_{i}}\left(T^{\dagger}, T\right) & =\frac{1}{\left(T^{\dagger}+T\right)^{n_{P}}} .
\end{aligned}
$$

Choices of $n_{X}, n_{\Phi}, n_{P}, l_{i}$ depend on the location of the fields on the D3/D7 branes. Besides, universal $l_{i}=1$ are adopted in our scenario to keep gauge coupling unification, so the gauge fields should reside on the D7 brane.

The superpotential takes the most general form involving the KKLT setup [3], the messenger sectors $W_{M}$ and visible sector $W_{\overline{M S S M}}$

$$
W=\left(\omega_{0}-A e^{-a T}\right)+W_{M}+W_{\overline{M S S M}},
$$

where the first term is generated from the fluxes and the second term from non-perturbative effects, such as gaugino condensation or D3-instanton. Within $W_{M}$, interactions between messengers and MSSM fields can possibly arise which will be discussed subsequently. The modulus $T$, which is not fixed by the background flux, can be stabilized by non-perturbative gaugino condensation with its VEV satisfying

$$
a \Re\langle T\rangle \approx \ln \left(\frac{A}{\omega_{0}}\right) \approx \ln \left(\frac{M_{\mathrm{Pl}}}{m_{3 / 2}}\right) \approx 4 \pi^{2}
$$

up to $\mathcal{O}\left(\ln \left[M_{\mathrm{Pl}} / m_{3 / 2}\right]^{-1}\right)$. Boundary value of the soft SUSY breaking parameters at the GUT scale can be seen in [11]. 


\section{Analytical expressions of soft SUSY breaking parameters}

Mirage mediation can be seen as a typical mixed modulus-anomaly mediation SUSY breaking mechanism with each contribution of similar size. Adding a messenger sector will add additional gauge mediation contributions. Besides, upon the messenger thresholds, new Yukawa interactions involving the messengers could arise. Such interactions may cause new contributions to trilinear couplings and sfermion masses (As an example, see our previous work [40]). Additional deflection with Yukawa mediation can be advantageous in several aspects.

- The value of trilinear coupling $\left|A_{t}\right|$ can be increased by additional contributions involving the new Yukawa interactions. Larger value of $A_{t}$ is always welcome in MSSM and NMSSM not only to accommodate the $125 \mathrm{GeV}$ Higgs but also to reduce [41] the EW fine tuning [42-44] involved.

- As noted in $[40,45,46]$, pure gauge mediation contributions are not viable to generate either trilinear couplings $A_{\kappa}, A_{\lambda}$ or soft scalar masses $m_{S}^{2}$ for singlet superfields $S$ which are crucial to solve the mu-problem of NMSSM. Deflection with Yukawa interactions will readily solve such difficulty.

To take into account such Yukawa mediation contributions in soft SUSY breaking parameters, it is better to derive the most general results involving the deflection. There are two approaches to obtain the low energy SUSY spectrum in the (deflected) mirage type mediation scenario:

- In the first approach, the mixed modulus-anomaly mediation soft SUSY spectrum is given by their boundary values at the GUT scale [11]. Such a spectrum will receive additional contributions towards its RGE running to low energy scale, especially the threshold corrections related to the appearance of messengers $[47,48]$. The soft SUSY breaking parameters are obtained by combing numerical RGE evolutions with threshold corrections. In [47], following this approach, some analytical expressions of the soft SUSY spectrum, for example the gaugino masses, are given. General expressions of the soft scalar masses and trilinear couplings are not given explicitly except for some simplified cases.

- In the second approach which we will adopt, the soft SUSY spectrum at low energy scale is derived directly from the low energy effective action. We know that the SUGRA description in eq. (2.1) can be seen as a Wilsonian effective action after integrating out the complex structure moduli and dilaton field. After the pseudo-modulus acquires a VEV and determines the messenger threshold, the messenger sector can be integrated out to obtain a low energy effective action below the messenger threshold. So we anticipate the Kahler metric $Z_{\Phi}$ and gauge kinetic $f_{i}$ will depend non-trivially on the messenger threshold $M_{\text {mess }}^{2} / \phi^{\dagger} \phi$ and $M_{\text {mess }} / \phi$, respectively. The resulting soft SUSY spectrum below the messenger threshold can be derived from the wavefunction renormalization approach [49]. The main difficulty here is to find the boundary value dependencies of the wavefunction and gauge kinetic term. 
In this approach, the most general expressions for soft SUSY breaking parameters in deflected modulus-anomaly (mirage) mediation SUSY breaking mechanism are derived below. Ordinary mirage mediation results can be obtained by setting the deflection parameter ' $d$ ' to zero.

- The gaugino masses are given by

$$
M_{i}=-g_{i}^{2}\left(\frac{F_{T}}{2} \frac{\partial}{\partial T}-\frac{F_{\phi}}{2} \frac{\partial}{\partial \ln \mu}+\frac{d F_{\phi}}{2} \frac{\partial}{\partial \ln |X|}\right) f_{a}\left(T, \frac{\mu}{\phi}, \sqrt{\frac{X^{\dagger} X}{\phi^{\dagger} \phi}}\right) .
$$

- The trilinear terms are given by

$$
\begin{aligned}
A_{Y_{a b c}} & \equiv A_{a b c} / y_{a b c} \\
& =\frac{1}{2} \sum_{i=a, b, c}\left(F^{T} \frac{\partial}{\partial T}-F_{\phi} \frac{\partial}{\partial \ln \mu}+d F_{\phi} \frac{\partial}{\partial \ln |X|}\right) \ln \left[e^{-K_{0} / 3} Z_{i}(\mu, X, T)\right] .
\end{aligned}
$$

- The soft sfermion masses are given by

$$
\begin{aligned}
-m_{\mathrm{soft}}^{2}(\mu)= & \left|\frac{F_{T}}{2} \frac{\partial}{\partial T}-\frac{F_{\phi}}{2} \frac{\partial}{\partial \ln \mu}+\frac{d}{2} F_{\phi} \frac{\partial}{\partial \ln |X|}\right|^{2} \ln \left[e^{-K_{0} / 3} Z_{i}(\mu, X, T)\right] \\
= & \left(\frac{\left|F_{T}\right|^{2}}{4} \frac{\partial^{2}}{\partial T \partial T^{*}}+\frac{F_{\phi}^{2}}{4} \frac{\partial^{2}}{\partial(\ln \mu)^{2}}+\frac{d^{2} F_{\phi}^{2}}{4} \frac{\partial^{2}}{\partial(\ln |X|)^{2}}-\frac{F_{T} F_{\phi}}{2} \frac{\partial^{2}}{\partial T \partial \ln \mu}\right. \\
& \left.+\frac{d F_{T} F_{\phi}}{2} \frac{\partial^{2}}{\partial T \partial \ln |X|}-\frac{d F_{\phi}^{2}}{2} \frac{\partial^{2}}{\partial \ln |X| \partial \ln \mu}\right) \ln \left[e^{-K_{0} / 3} Z_{i}(\mu, X, T)\right] .
\end{aligned}
$$

From the previous general expressions, we can deduce the concrete analytical results for soft SUSY parameters. In our notation, we define the modulus mediation part

$$
M_{0} \equiv \frac{F_{T}}{T+T^{*}}, \quad q_{Y_{i j k}} \equiv 3-\left(n_{i}+n_{j}+n_{k}\right) .
$$

The gauge and Yukawa couplings are used in the form

$$
\alpha_{i}=\frac{g_{i}^{2}}{4 \pi}, \quad \alpha_{\lambda_{i j k}}=\frac{\lambda_{i j k}^{2}}{4 \pi} .
$$

\subsection{Gaugino mass}

The gaugino mass below the messenger scale can be obtained from eq. (3.1). At the GUT (compactification scale) $M_{G}$, the gauge coupling unification requires

$$
T^{l_{a}}=\frac{1}{g^{2}(\mathrm{GUT})}
$$

The gauge coupling at scale $\mu$ just below the messenger threshold $M$ is given as

$$
\begin{aligned}
\frac{1}{g_{i}^{2}(\mu)} & =\frac{1}{g_{i}^{2}(\mathrm{GUT})}+\frac{b_{i}+\Delta b_{i}}{8 \pi^{2}} \ln \frac{M_{G}}{|X|}+\frac{b_{i}}{8 \pi^{2}} \ln \frac{|X|}{\mu}, \\
& =T^{l_{a}}+\frac{b_{i}+\Delta b_{i}}{8 \pi^{2}} \ln \frac{M_{G}}{M}+\frac{b_{i}}{8 \pi^{2}} \ln \frac{M}{\mu} .
\end{aligned}
$$


The derivatives are given as

$$
\frac{\partial}{\partial \ln \mu}\left(\frac{1}{g_{i}^{2}(\mu)}\right)=-\frac{b_{i}}{8 \pi^{2}}, \quad \frac{\partial}{\partial \ln M}\left(\frac{1}{g_{i}^{2}(\mu)}\right)=-\frac{\Delta b_{i}}{8 \pi^{2}},
$$

and

$$
\frac{\partial}{\partial T}\left(\frac{1}{g_{a}^{2}(\mu)}\right)=l_{a} T^{l_{a}-1} \Longrightarrow-2 \frac{1}{g_{a}^{3}} \frac{\partial g_{a}(\mu)}{\partial T}=l_{a} T^{l_{a}-1}
$$

So we can obtain the analytical results for gaugino mass

$$
M_{i}(\mu)=g_{i}^{2}(\mu)\left[l_{a} \frac{F_{T}}{2 T} \frac{1}{g_{a}^{2}(\mathrm{GUT})}+\frac{F_{\phi}}{2} \frac{b_{i}}{8 \pi^{2}}-\frac{d}{2} F_{\phi} \frac{\Delta b_{i}}{8 \pi^{2}}\right],
$$

with $\Delta b_{i} \equiv b_{i}^{\prime}-b_{i}$ and $b_{i}^{\prime}, b_{i}$ the gauge beta function upon and below the messenger thresholds, respectively. This results can coincide with the gaugino masses predicted from RGE running with threshold corrections at the messenger scale. Following the approach in [26-28], the gaugino mass at the scale $\mu$ slightly below the messenger scale $M$ will receive additional gauge mediation contributions

$$
\begin{aligned}
M_{i}(\mu \lesssim M) & =\frac{g_{i}^{2}(M)}{g_{i}^{2}(\mathrm{GUT})} M_{i}(\mathrm{GUT})-F_{\phi} \frac{g_{i}^{2}(M)}{16 \pi^{2}}(d+1) \Delta b_{i} \\
& =g_{i}^{2}(M)\left[l_{a} \frac{F_{T}}{2 T} \frac{1}{g_{a}(\mathrm{GUT})}+\frac{F_{\phi}}{2} \frac{b_{i}+\Delta b_{i}}{8 \pi^{2}}\right]-F_{\phi} \frac{g_{i}^{2}(M)}{16 \pi^{2}}(d+1) \Delta b_{i}
\end{aligned}
$$

with

$$
M_{i}(\mathrm{GUT})=g_{i}^{2}(\mathrm{GUT})\left[l_{a} \frac{F_{T}}{2 T} \frac{1}{g_{a}^{2}(\mathrm{GUT})}+\frac{F_{\phi}}{2} \frac{b_{i}+\Delta b_{i}}{8 \pi^{2}}\right]
$$

Then we can obtain the gaugino mass at scale $\mu<M$ from one-loop RGE

$$
\begin{aligned}
M_{i}(\mu) & =\frac{g_{i}^{2}(\mu)}{g_{i}^{2}(M)} M_{i}(\mu \lesssim \ln M) \\
& =g_{i}^{2}(\mu)\left[l_{a} \frac{F_{T}}{2 T} \frac{1}{g_{a}^{2}(\mathrm{GUT})}+\frac{F_{\phi}}{2} \frac{b_{i}}{8 \pi^{2}}\right]-F_{\phi} \frac{g_{i}^{2}(\mu)}{16 \pi^{2}} d \Delta b_{i}
\end{aligned}
$$

So we can see that the two results agree with each other.

\subsection{Trilinear terms}

From the form of wavefunction

$$
Z_{i}(\mu)=Z_{i}(\Lambda) \prod_{l=y_{t}, y_{b}, y_{\tau}}\left(\frac{y_{l}(\mu)}{y_{l}(\Lambda)}\right)^{A_{l}} \prod_{k=1,2,3}\left(\frac{g_{k}(\mu)}{g_{k}(\Lambda)}\right)^{B_{k}}
$$

we can obtain the trilinear terms for scales below the messenger $M$ from eq. (3.2). The main challenge is the calculation of $\partial Z_{i} / \partial T$. 
Before we derive the final results involving all $y_{t}, y_{b}, y_{\tau}$ and $g_{3}, g_{2}, g_{1}$, we will study first the simplest case in which only the top Yukawa $\alpha_{t} \equiv y_{t}^{2} / 4 \pi$ and $\alpha_{s} \equiv g_{3}^{2} / 4 \pi$ are kept in the anomalous dimension. The RGE equation for $\alpha_{t}$ and $\alpha_{s}$ takes the form

$$
\frac{d}{d t} \ln \alpha_{t}=\frac{1}{\pi}\left(3 \alpha_{t}-\frac{8}{3} \alpha_{s}\right), \quad \frac{d}{d t} \ln \alpha_{s}=-\frac{1}{2 \pi} b_{3} \alpha_{s}
$$

Note the definition $b_{3}$ differs by a minus sign. Define $A=\ln \left(\alpha_{t} \alpha_{s}^{-\frac{16}{3 b_{3}}}\right)$, the equation can be written as

$$
\frac{d}{d t} e^{-A}=-\frac{3}{\pi} \alpha_{s}^{\frac{16}{3 b_{3}}}
$$

So we can exactly solve the differential equation to get

$$
\left[\frac{\alpha_{t}(\mu)}{\alpha_{t}(\Lambda)}\left(\frac{\alpha_{s}(\mu)}{\alpha_{s}(\Lambda)}\right)^{-\frac{16}{3 b_{3}}}\right]^{-1}=1-\frac{3 \alpha_{t}(\Lambda)}{\pi} \frac{2 \pi}{\frac{16}{3}-b_{3}}\left[\alpha_{s}(\Lambda)^{-1}-\left(\frac{\alpha_{s}(\mu)}{\alpha_{s}(\Lambda)}\right)^{\frac{16}{3 b_{3}}} \alpha_{s}^{-1}(\mu)\right] .
$$

Expanding the expressions and neglect high order terms, we finally have

$$
\frac{\partial}{\partial T}\left[\ln \alpha_{t}(\mu)-\ln \alpha_{t}(\Lambda)\right] \approx \frac{\partial}{\partial T}\left[-\frac{8}{3 \pi} \alpha_{s}(\mu)+\frac{3}{\pi} \alpha_{t}(\mu)\right] \ln \left(\frac{\Lambda}{\mu}\right),
$$

after calculations. It can be observed that the expression within the square bracket is just the beta function of top Yukawa coupling.

Now we will calculate $\partial Z_{i} / \partial T$ with all $y_{t}, y_{b}, y_{\tau}$ and $g_{3}, g_{2}, g_{1}$ taking into account in the expression.

- Deduction of $\partial Z_{i} / \partial T$ without messenger deflections.

From the form of wavefunction

$$
Z_{i}(\mu)=Z_{i}\left(M_{G}\right) \prod_{l=y_{t}, y_{b}, y_{\tau}}\left(\frac{y_{l}(\mu)}{y_{l}\left(M_{G}\right)}\right)^{A_{l}} \prod_{k=1,2,3}\left(\frac{g_{k}(\mu)}{g_{k}\left(M_{G}\right)}\right)^{B_{k}}
$$

and renormalizatoin $Z=Z_{0}(1+\delta Z)$, we have

$$
\begin{aligned}
\frac{\partial \ln e^{-K_{0} / 3} Z_{i}}{\partial T} & =\frac{\partial}{\partial T} \ln e^{-K_{0} / 3} Z_{i}\left(M_{G}\right)+\frac{\partial}{\partial T} \delta Z_{i} \\
& =\frac{1-n_{i}}{T}+\sum_{m=1,2}\left[\sum_{a} \frac{\partial g_{a ; m}}{\partial T} \frac{\partial \delta Z_{i}}{\partial g_{a ; m}}+\sum_{a, b, c} \frac{\partial \ln y_{a b c ; m}}{\partial T} \frac{\partial \delta Z_{i}}{\partial \ln y_{a b c ; m}}\right]
\end{aligned}
$$

with $m=1,2$ corresponding to the value at the scale $\mu$ and the GUT scale, respectively. The derivative with respect to $g_{m}$ gives

$$
\frac{\partial \ln e^{-K_{0} / 3} Z_{i}}{\partial g_{m}(\mu)}=\frac{B_{m}}{g_{m}(\mu)}, \quad \frac{\partial \ln e^{-K_{0} / 3} Z_{i}}{\partial g_{m}\left(M_{G}\right)}=-\frac{B_{m}}{g_{m}\left(M_{G}\right)},
$$


and

$$
\frac{\partial g_{i}(\mu)}{\partial T}=-\frac{l_{a} T^{l_{a}-1}}{2} g_{i}^{3}(\mu), \quad \frac{\partial g_{i}\left(M_{G}\right)}{\partial T}=-\frac{l_{a} T^{l_{a}-1}}{2} g_{i}^{3}\left(M_{G}\right) .
$$

The derivative with respect to $y_{l}$ gives

$$
\frac{\partial \ln e^{-K_{0} / 3} Z_{i}}{\partial y_{l}(\mu)}=\frac{A_{l}}{y_{l}(\mu)}, \quad \frac{\partial \ln e^{-K_{0} / 3} Z_{i}}{\partial y_{l}\left(M_{G}\right)}=-\frac{A_{l}}{y_{l}\left(M_{G}\right)},
$$

and

$$
\frac{\partial y_{l}\left(M_{G}\right)}{\partial T}=-\frac{y_{l}\left(M_{G}\right)}{2}\left[\frac{3-a_{i j k}}{T}\right], \quad \frac{\partial \ln \alpha_{Y_{a b c}}(\mu)}{\partial T}=-\left[\frac{3-a_{i j k}}{T}\right] .
$$

From the beta function of the Yukawa couplings, we have

$$
\begin{aligned}
\frac{\partial \ln \alpha_{Y_{a b c}}(\mu)}{\partial T} & =\frac{\partial \ln \alpha_{Y_{a b c}}\left(M_{G}\right)}{\partial T}-\frac{\partial}{\partial T} \int_{\mu}^{M_{G}}\left(\frac{d}{d \ln \mu^{\prime}} \ln \alpha_{Y_{a b c}}\right) d \ln \mu^{\prime}, \\
& =-\frac{3-a_{a b c}}{T}-\frac{1}{2 \pi} \int_{\mu}^{M_{G}} d \ln \mu^{\prime}\left(\sum_{Y_{l m n}} c_{l m n} \frac{\partial}{\partial T} \alpha_{Y_{l m n}}\left(\mu^{\prime}\right)+\sum_{m} d_{m} \frac{\partial}{\partial T} \alpha_{m}\left(\mu^{\prime}\right)\right), \\
& \approx-\frac{3-a_{a b c}}{T}+\frac{1}{2 \pi}\left[\sum_{Y_{l m n}} c_{l m n} \frac{3-a_{l m n}}{T} \alpha_{Y_{l m n}}(\mu)+\sum_{m} d_{m} \frac{l_{a}}{T} \frac{\alpha_{m}^{2}(\mu)}{\alpha_{m}\left(M_{G}\right)}\right] \ln \left(\frac{M_{G}}{\mu}\right),
\end{aligned}
$$

with $a_{a b c}=n_{a}+n_{b}+n_{c}$.

So the derivative with respect to $T$ is given by

$$
\begin{aligned}
\frac{\partial}{\partial T} \delta Z_{i}= & \sum_{m=1,2}\left[\sum_{a} \frac{\partial \ln \alpha_{a ; m}}{\partial T} \frac{\partial \delta Z_{i}}{\partial \ln \alpha_{a ; m}}+\sum_{Y_{a b c}} \frac{\partial \ln \alpha_{Y_{a b c ; m}}}{\partial T} \frac{\partial \delta Z_{i}}{\partial \ln \alpha_{Y_{a b c} m}}\right], \\
= & \sum_{a} \frac{B_{a}}{2}\left[\frac{\partial}{\partial T} \ln \left(\frac{\alpha_{a}(\mu)}{\alpha_{a}(\Lambda)}\right)\right]+\sum_{Y_{a b c}} \frac{A_{Y_{a b c}}}{2}\left[\frac{\partial}{\partial T} \ln \left(\frac{\alpha_{Y_{a b c}}(\mu)}{\alpha_{Y_{a b c}}(\Lambda)}\right)\right], \\
\approx & \sum_{a} \frac{B_{a}}{2}\left[\frac{\partial}{\partial T}\left(-\frac{b_{a}}{2 \pi} \alpha_{a}(\mu) \ln \left(\frac{\Lambda}{\mu}\right)\right)\right] \\
& +\sum_{Y_{a b c}} \frac{A_{Y_{a b c}}}{2} \frac{1}{2 \pi}\left[\sum_{Y_{l m n}} c_{l m n} \frac{3-a_{l m n}}{T} \alpha_{Y_{l m n}}(\mu)-\sum_{m} d_{m} \frac{\partial}{\partial T} \alpha_{m}(\mu)\right] \ln \left(\frac{\Lambda}{\mu}\right) .
\end{aligned}
$$

We know from the expression of the wavefunction, the coefficients satisfy

$$
\sum_{Y_{a b c}} \frac{A_{Y_{a b c}}}{2} d_{m}+b_{m} \frac{B_{m}}{2}=-\frac{\partial G_{Z_{i}}}{\partial \alpha_{m}},
$$


for coefficients of $\alpha_{m}$. While the coefficients for Yukawa couplings $Y_{l m n}$ within $Z_{i}$ satisfy

$$
\sum_{Y_{a b c}} \frac{A_{Y_{a b c}}}{2} c_{l m n}=-\frac{\partial G_{Z_{i}}}{\partial \alpha_{Y_{l m n}}} .
$$

So the final results reduces to

$$
\begin{aligned}
\frac{\partial}{\partial T} \ln e^{-K_{0} / 3} Z_{i} & \approx-\frac{1}{2 \pi}\left[\frac{d_{j k}^{i}}{2} \frac{3-a_{Y_{i j k}}}{T} \alpha_{Y_{i j k}}(\mu)-2 C_{a}(i) \frac{l_{a}}{T} \alpha_{a}(\mu)\right] \ln \left(\frac{\mathrm{GUT}}{\mu}\right)+\frac{1-n_{i}}{T} \\
& =\frac{1}{2 \pi} \frac{\partial}{\partial T}\left[\frac{d_{j k}^{i}}{2} \alpha_{Y_{i j k}}(\mu)-2 C_{a}(i) \alpha_{a}(\mu)\right] \ln \left(\frac{\mathrm{GUT}}{\mu}\right)+\frac{1-n_{i}}{T}
\end{aligned}
$$

with the expressions in the second square bracket being the anomalous dimension of $Z_{i}$.

- Deduction of $\partial Z_{i} / \partial T$ with messenger deflections.

The form of wavefunction should be

$$
\begin{gathered}
Z_{i}(\mu)=Z_{i}\left(M_{G}\right) \prod_{l=y_{t}, y_{b}, y_{\tau}}\left(\frac{y_{l}(M)}{y_{l}\left(M_{G}\right)}\right)^{A_{l}} \prod_{k=1,2,3}\left(\frac{g_{k}(M)}{g_{k}\left(M_{G}\right)}\right)^{B_{k}} \prod_{k=y_{U}}\left(\frac{y_{k}(M)}{y_{k}\left(M_{G}\right)}\right)^{C_{k}} \\
\prod_{l=y_{t}, y_{b}, y_{\tau}}\left(\frac{y_{l}(\mu)}{y_{l}(M)}\right)^{A_{l}^{\prime}} \prod_{k=1,2,3}\left(\frac{g_{k}(\mu)}{g_{k}(M)}\right)^{B_{k}^{\prime}}
\end{gathered}
$$

with $y_{U}$ the interaction involving the messengers which will be integrated below the messenger scale.

We have

$$
\begin{aligned}
\frac{\partial \ln e^{-K_{0} / 3} Z_{i}}{\partial T}= & \frac{\partial}{\partial T} \ln e^{-K_{0} / 3} Z_{i}^{0}+\frac{\partial}{\partial T} \delta Z_{i}, \\
= & {\left[\sum_{g_{a}} \frac{\partial \ln \left(\frac{g_{a}(\mu)}{g_{a}(M)}\right)}{\partial T} \frac{\partial \delta Z_{i}}{\partial \ln \left(\frac{g_{a}(\mu)}{g_{a}(M)}\right)}+\sum_{y_{a b c}} \frac{\partial \ln \left(\frac{y_{a b c}(\mu)}{y_{a b c}(M)}\right)}{\partial T} \frac{\partial \delta Z_{i}}{\partial \ln \left(\frac{y_{a b c}(\mu)}{y_{a b c}(M)}\right)},\right.} \\
& +\sum_{g_{a}} \frac{\partial \ln \left(\frac{g_{a}(M)}{g_{a}\left(M_{G}\right)}\right)}{\partial T} \frac{\partial \delta Z_{i}}{\partial \ln \left(\frac{g_{a}(M)}{g_{a}\left(M_{G}\right)}\right)}+\sum_{y_{a b c}} \frac{\partial \ln \left(\frac{y_{a b c}(M)}{y_{a b c}\left(M_{G}\right)}\right)}{\partial T} \frac{\partial \delta Z_{i}}{\partial \ln \left(\frac{y_{a b c}(M)}{y_{a b c}\left(M_{G}\right)}\right)}, \\
& \left.+\sum_{y_{U}} \frac{\partial \ln \left(\frac{y_{U}(M)}{y_{U}\left(M_{G}\right)}\right)}{\partial T} \frac{\partial \delta Z_{i}}{\partial \ln \left(\frac{y_{U}(M)}{y_{U}\left(M_{G}\right)}\right)}\right]+\frac{1-n_{i}}{T},
\end{aligned}
$$

with $m=1,2$ corresponding to the value at the scale $\mu$ and the GUT scale, respectively. 
Using similar deductions for Yukawa couplings, we can obtain

$$
\begin{aligned}
& \frac{\partial \ln e^{-K_{0} / 3} Z_{i}}{\partial T} \\
= & \frac{1-n_{i}}{T}-\frac{1}{4 \pi} \sum_{g_{a}}\left(B_{a} b_{a}^{\prime} \frac{\partial \alpha_{a}(M)}{\partial T} \ln \left(\frac{M_{G}}{M}\right)+B_{a}^{\prime} b_{a} \frac{\partial \alpha_{a}(M)}{\partial T} \ln \left(\frac{M}{\mu}\right)\right) \\
& +\sum_{y_{a b c} \in y_{t}, y_{b}, y_{\tau}} A_{y_{a b c}} \ln \left(\frac{M_{G}}{M}\right) \frac{1}{4 \pi}\left[\sum_{Y_{l m n} \in y_{t}, y_{b}, y_{\tau}} c_{l m n} \frac{3-a_{l m n}}{T} \alpha_{Y_{l m n}}(M),\right. \\
& \left.+\sum_{Y_{l m n} \in y_{U}} \tilde{c}_{l m n} \frac{3-a_{U}}{T} \alpha_{Y_{U}}(M)-\sum_{g_{m}} d_{m} \frac{\partial}{\partial T} \alpha_{m}(M)\right] \cdot \\
& +\sum_{y_{a b c} \in y_{t}, y_{b}, y_{\tau}} A_{y_{a b c}}^{\prime} \ln \left(\frac{\mu}{M}\right) \frac{1}{4 \pi}\left[\sum_{Y_{l m n} \in y_{t}, y_{b}, y_{\tau}} c_{l m n} \frac{3-a_{l m n}}{T} \alpha_{Y_{l m n}}(M)-\sum_{g_{m}} d_{m} \frac{\partial}{\partial T} \alpha_{m}(M)\right] . \\
& +\sum_{y_{U}} C_{y_{U}} \ln \left(\frac{M}{M_{G}}\right) \frac{1}{4 \pi}\left[\sum_{\tilde{Y}_{l m n} \in y_{t}, y_{b}, y_{\tau}} d_{l m n} \frac{3-a_{l m n}}{T} \alpha_{Y_{l m n}}(M)-\sum_{g_{m}} f_{m} \frac{\partial}{\partial T} \alpha_{m}(M),\right. \\
& \left.+\sum_{\tilde{Y}_{l m n} \in y_{U}} \tilde{d}_{l m n} \frac{3-a_{U}}{T} \alpha_{Y_{U}}(M)\right],
\end{aligned}
$$

with the beta function for $y_{t}, y_{b}, y_{\tau}$ Yukawa couplings

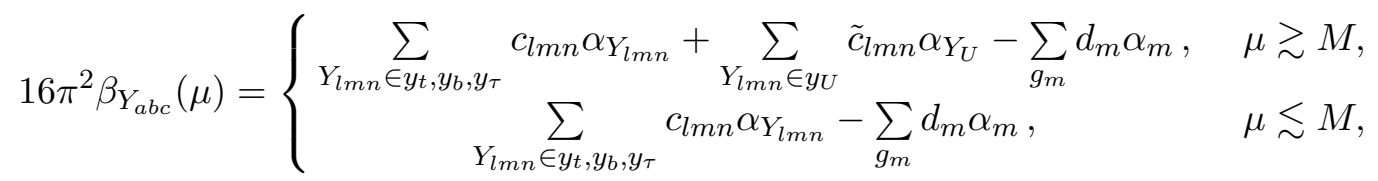

and the beta function for new messenger-matter $y_{U}$ Yukawa couplings

$$
16 \pi^{2} \beta_{Y_{U}}=\sum_{\tilde{Y}_{l m n} \in y_{t}, y_{b}, y_{\tau}} d_{l m n} \alpha_{\tilde{Y}_{l m n}}+\sum_{\tilde{Y}_{l m n} \in y_{U}} \tilde{d}_{l m n} \alpha_{Y_{U}}-\sum_{m} f_{m} \alpha_{m}
$$

The coefficients satisfy

$$
\begin{aligned}
\sum_{Y_{a b c}}\left(A_{y_{a b c}}-A_{y_{a b c}}^{\prime}\right) c_{l m n}+\sum_{Y_{U}} C_{y_{U}} d_{l m n} & \left.=0, \quad \text { (for } y_{t}, y_{b}, y_{\tau} \text { coefficients }\right) \\
\sum_{Y_{a b c}} A_{y_{a b c}} \tilde{c}_{l m n}+\sum_{Y_{U}} C_{y_{U}} \tilde{d}_{l m n} & =0, \quad \text { (for yu coefficients) } \\
B_{m} b_{m}^{\prime}+\sum_{Y_{a b c}} A_{y_{a b c}} d_{m}+\sum_{Y_{U}} C_{Y_{U}} f_{m} & =B_{m}^{\prime} b_{m}+\sum_{Y_{a b c}} A_{y_{a b c}}^{\prime} d_{m}
\end{aligned}
$$

and similarly for $g_{m}$, the sum then reduces to the previous case. So we have for $\mu<M$

$$
\begin{aligned}
& \frac{\partial}{\partial T} \ln \left(e^{-K_{0} / 3} Z_{i}(\mu)\right)-\frac{1-n_{i}}{T}, \\
& \quad \approx-\frac{1}{2 \pi}\left[\frac{1}{2} d_{j k}^{i} \frac{3-a_{Y_{i j k}}}{T} \alpha_{Y_{i j k}}(\mu)-2 C_{a}(i) \frac{l_{a}}{T} \alpha_{a}(\mu)\right] \ln \left(\frac{M_{G}}{\mu}\right) .
\end{aligned}
$$


Note that the expressions within the square bracket agree with the anomalous dimension of $Z_{i}^{-}$below the messenger threshold $M$

$$
G_{i}^{-} \equiv \frac{d Z_{i}^{-}}{d \ln \mu} \equiv-\frac{1}{8 \pi^{2}}\left(\frac{1}{2} d_{k l}^{i} \lambda_{i k l}^{2}-2 c_{r}^{i} g_{r}^{2}\right)
$$

The $G_{i}^{+}$, which is the anomalous dimension of $Z_{i}$ upon the messenger threshold $M$, do not appear in the final expressions.

The dependence of $Z_{i}$ on messenger scale $M$ can be derived following the techniques [50, 51] developed in gauge mediated SUSY breaking (GMSB) [52-58] scenarios. From the expressions of the wavefunction, we can obtain

$$
\begin{aligned}
\frac{\partial}{\partial \ln M} \ln \left[e^{-K_{0} / 3} Z_{i}\right]= & \frac{1}{4 \pi} \sum_{g_{k}}\left[\left(B_{k}-B_{k}^{\prime}\right)\left(b_{k}+N_{F}\right) \alpha_{k}(M)+B_{k}^{\prime} N_{F} \alpha_{k}(\mu)\right] \\
& +\sum_{Y_{l}}\left[\left(A_{l}-A_{l}^{\prime}\right) G_{Y_{l}}^{+}(\ln M)+A_{l}^{\prime} \frac{\partial Y_{l}(\ln \mu, M)}{\partial \ln M}\right]+\sum_{Y_{U}}\left[C_{l} G_{Y_{U}}^{+}(\ln M)\right] .
\end{aligned}
$$

So the main challenge is to calculate $\partial \ln Y_{a}(\mu, \ln M) / \partial \ln M$.

From the beta functions for Yukawa couplings upon and below the messenger thresholds, the Yukawa couplings at scale $\mu<M$ is given as

$$
\ln Y_{a}(\mu, \ln M)=\ln Y_{a}\left(M_{G}\right)+\int_{M_{G}}^{\ln M} G_{Y_{a}}^{+}\left(t^{\prime}\right) d t^{\prime}+\int_{\ln M}^{\ln \mu} G_{Y_{a}}^{-}\left(t^{\prime}, \ln M\right) d t^{\prime}
$$

with the Yukawa beta functions expressed as

$$
\begin{aligned}
\beta_{Y_{a}} \equiv G_{Y_{a}} & \equiv-\frac{1}{2} \sum_{i \in a} G^{i} \equiv \frac{1}{4 \pi}\left(\frac{1}{2} \tilde{d}_{k l}^{i} \alpha_{\lambda_{i k l}}-2 \tilde{c}_{r} \alpha_{r}\right), \\
G_{i}=\frac{d \ln Z_{i}}{d \ln \mu} & \equiv-\frac{1}{2 \pi}\left(\frac{1}{2} d_{k l}^{i} \alpha_{\lambda_{i k l}}-2 c_{r}^{i} \alpha_{r}\right) .
\end{aligned}
$$

We can derive the Yukawa couplings dependence on ' $\ln M^{\prime}$ at scale $\mu<M$

$$
\begin{aligned}
\frac{\partial}{\partial \ln M} \ln Y_{a}(\mu, \ln M) & =\left[G_{Y_{a}}^{+}(\ln M)-G_{Y_{a}}^{-}(\ln M, \ln M)\right]+\int_{\ln M}^{\ln \mu} \frac{\partial}{\partial \ln M} G_{Y_{a}}^{-}\left(t^{\prime}, \ln M\right) d t^{\prime} \\
& \approx \Delta G_{Y_{a}}(\ln M)-\frac{1}{16 \pi^{2}}\left[\tilde{d}_{k l}^{i} \lambda_{i k l}(\mu) \Delta G_{\lambda_{i k l}}-4 \tilde{c}_{r} \frac{\Delta b_{r}}{16 \pi^{2}} g_{r}^{4}(\mu)\right] \ln \left(\frac{M}{\mu}\right) .
\end{aligned}
$$

In the case $\Delta G=0$ in which no additional Yukawa couplings involving the messengers are present, we have

$$
\frac{\partial}{\partial \ln M} \ln Y_{a}(\mu, \ln M) \approx \frac{\tilde{c}_{r}}{4 \pi^{2}} \Delta b_{r} \alpha_{r}^{2}(\mu) \ln \left(\frac{M}{\mu}\right)
$$


Note that at the messenger scale

$$
\frac{\partial}{\partial \ln M} \ln Y_{a}(\ln M, \ln M)=\Delta G_{a}(\ln M)
$$

The expressions takes a simple form at the scale $\mu$ slightly below the messenger scale $M$

$$
\begin{aligned}
& A_{Y_{a b c}}(\mu \lesssim M)-\left(3-a_{a b c}\right) \frac{F_{T}}{T+T^{*}} \\
& =\sum_{l=a, b, c}\left\{-\frac{F_{T}}{T+T^{*}} \frac{1}{2 \pi}\left[\frac{1}{2} d_{j k}^{i}\left(3-a_{Y_{i j k}}\right) \alpha_{Y_{i j k}}(\mu)-2 C_{a}(i) l_{a} \alpha_{a}(\mu)\right] \ln \left(\frac{\mathrm{GUT}}{\mu}\right)\right. \\
& \left.\quad+d F_{\phi} \frac{\Delta G_{i}}{2}-\frac{F_{\phi}}{2} G_{i}^{-}\right\},
\end{aligned}
$$

with $\Delta G_{i} \equiv G_{i}^{+}-G_{i}^{-}$[here ${ }^{\prime} G_{i}^{+}\left(G_{i}^{-}\right)^{\prime}$ denotes respectively the anomalous dimension of $Z_{i}$ upon (below) the messenger threshold] the discontinuity of anomalous dimension across the messenger threshold.

\subsection{Soft scalar masses}

The soft scalar masses are given as

$$
\begin{aligned}
-m_{\text {soft }}^{2}= & \left|\frac{F_{T}}{2} \frac{\partial}{\partial T}-\frac{F_{\phi}}{2} \frac{\partial}{\partial \ln \mu}+d F_{\phi} \frac{\partial}{\partial \ln X}\right|^{2} \ln \left[e^{-K_{0} / 3} Z_{i}(\mu, X, T)\right], \\
= & \left(\frac{\left|F_{T}\right|^{2}}{4} \frac{\partial^{2}}{\partial T \partial T^{*}}+\frac{F_{\phi}^{2}}{4} \frac{\partial^{2}}{\partial(\ln \mu)^{2}}+\frac{d^{2} F_{\phi}^{2}}{4} \frac{\partial}{\partial(\ln |X|)^{2}}-\frac{F_{T} F_{\phi}}{2} \frac{\partial^{2}}{\partial T \partial \ln \mu}\right. \\
& \left.+\frac{d F_{T} F_{\phi}}{2} \frac{\partial^{2}}{\partial T \partial \ln |X|}-\frac{d F_{\phi}^{2}}{2} \frac{\partial^{2}}{\partial \ln |X| \partial \ln \mu}\right) \ln \left[e^{-K_{0} / 3} Z_{i}(\mu, X, T)\right] .
\end{aligned}
$$

The new ingredients are the second derivative of $Z_{i}$ with respect to $T$

$$
\begin{aligned}
& \frac{\partial^{2}}{\partial T^{2}} \ln \left[e^{-3 K_{0}} Z_{i}\right] \\
& =-\frac{1}{2 \pi} \frac{\partial}{\partial T}\left[\frac{1}{2} d_{j k}^{i} \frac{3-a_{Y_{i j k}}}{T} \alpha_{Y_{i j k}}(\mu)-2 C_{a}(i) \frac{l_{a}}{T} \alpha_{a}(\mu)\right] \ln \left(\frac{\mathrm{GUT}}{\mu}\right)-\frac{1-n_{i}}{T^{2}} \\
& =-\frac{1}{2 \pi}\left[\frac{1}{2} d_{j k}^{i} \frac{3-a_{Y_{i j k}}}{T} \alpha_{Y_{i j k}}(\mu)\left[-\frac{3-a_{Y_{i j k}}}{T}+\frac{1}{2 \pi}\left(\frac{\tilde{d}_{m n}^{p}}{2} \frac{3-a_{Y_{i j k}}}{T} \alpha_{Y_{m n p}}-2 c_{r} \frac{l_{a}}{T} \alpha_{a}\right) \ln \left(\frac{\mathrm{GUT}}{\mu}\right)\right]\right. \\
& \left.-\frac{1}{2} d_{j k}^{i} \frac{\left(3-a_{Y_{i j k}}\right)}{T^{2}} \alpha_{Y_{i j k}}(\mu)-2 C_{a}(i)\left(-\frac{l_{a}}{T^{2}} \alpha_{a}(\mu)-\frac{l_{a}^{2}}{T^{2}} \frac{\alpha_{a}^{2}(\mu)}{\alpha_{a}(\mathrm{GUT})}\right)\right] \ln \left(\frac{\mathrm{GUT}}{\mu}\right)-\frac{1-n_{i}}{T^{2}},
\end{aligned}
$$

with the beta function of $Y_{i j k}$ given by

$$
\frac{d \ln Y_{i j k}}{d \ln \mu}=\frac{1}{16 \pi^{2}}\left[\frac{\tilde{d}_{m n}^{p}}{2} \alpha_{Y_{m n p}}-2 c_{r}^{i} \alpha_{i}\right],
$$


and

$$
\frac{\alpha_{a}(\mu)}{\alpha_{a}(\mathrm{GUT})}=1-\frac{b_{a}}{2 \pi} \alpha_{a}(\mu) \ln \left(\frac{\mathrm{GUT}}{\mu}\right) .
$$

The other terms within eq. (3.40) can be found in GMSB (not involving $\partial T$ ) or calculated directly using eq. (3.21) and eq. (3.23) (involving $\partial T$ ). We list the analytical results of deflected mirage mediation in appendix $\mathrm{B}$.

\section{Applications of the general analytical results}

\subsection{Analytical results for mirage mediation}

Equipped with the previous deduction, we can readily reproduce the ordinary mirage mediation results by setting $d \rightarrow 0$. As the visible gauge fields originate from $\mathrm{D} 7$ branes and gauge coupling unification is always assumed, we adopt $l_{a}=1$. The following definitions are used

$$
M_{0} \equiv \frac{F_{T}}{2 T} \equiv \frac{F_{\phi}}{\alpha \ln \left(\frac{M_{\mathrm{Pl}}}{m_{3 / 2}}\right)} \approx \frac{F_{\phi}}{4 \pi^{2} \alpha},
$$

with the parameter $\alpha$ defined as the ratio between the anomaly mediation and modulus mediation contributions and the approximation $\ln \left(M_{\mathrm{Pl}} / m_{3 / 2}\right) \approx 4 \pi^{2}$. We have

- Gaugino mass:

$$
\begin{aligned}
M_{i}(\mu) & =l_{a} M_{0} \frac{g_{i}^{2}(\mu)}{g_{a}^{2}(\mathrm{GUT})}+\frac{F_{\phi}}{16 \pi^{2}} b_{i} g_{i}^{2}(\mu), \\
& =l_{a} M_{0}\left[1-\frac{b_{i}}{8 \pi^{2}} g_{i}^{2}(\mu) \ln \frac{\mathrm{GUT}}{\mu}\right]+\frac{M_{0}}{4} \alpha b_{i} g_{i}^{2}(\mu) .
\end{aligned}
$$

So we can see that at the scale $\mu_{M i}$ which satisfies

$$
\frac{1}{8 \pi^{2}} \ln \left(\frac{M_{\mathrm{GUT}}}{\mu_{M i}}\right)=\frac{\alpha}{4},
$$

the gaugino masses unify at such 'mirage' unification scale

$$
\mu_{M i}=M_{\mathrm{GUT}} e^{-2 \alpha \pi^{2}} \approx M_{\mathrm{GUT}}\left(\frac{m_{3 / 2}}{M_{\mathrm{Pl}}}\right)^{\frac{\alpha}{2}} .
$$

- Trilinear term:

$$
\begin{aligned}
& A_{Y_{a b c}}(\mu \lesssim M) \\
& =\sum_{l=a, b, c}\left\{-M_{0} \frac{1}{2 \pi}\left[\frac{1}{2} d_{j k}^{i}\left(3-a_{Y_{i j k}}\right) \alpha_{Y_{i j k}}(\mu)-2 C_{a}(i) l_{a} \alpha_{a}(\mu)\right] \ln \left(\frac{\mathrm{GUT}}{\mu}\right)\right. \\
& \quad+\frac{F_{\phi}}{4 \pi}\left[\frac{1}{2} d_{j k}^{i} \alpha_{Y_{i j k}}(\mu)-2 C_{a}(i) \alpha_{a}(\mu)\right]+\left(3-a_{a b c}\right) M_{0} .
\end{aligned}
$$


In case the effect of Yukawa couplings are negligible or $a_{Y_{i j k}}=2$, the trilinear term also "unify" at a mirage scale at which the last two terms cancel

$$
\frac{1}{2 \pi} \ln \left(\frac{M_{\mathrm{GUT}}}{\mu_{M i}}\right)=\pi \alpha,
$$

which is just the mirage scale for gaugino mass "unification".

- Soft scalar masses:

$$
\begin{aligned}
-m_{i}^{2} & =\frac{M_{0}^{2}}{2 \pi} \ln \left(\frac{M_{\mathrm{GUT}}}{\mu}\right)\left\{\frac{d_{j k}^{i}}{2}\left(q_{Y_{i j k}}^{2}+q_{Y_{i j k}}\right) \alpha_{Y_{i j k}}(\mu)-2 C_{a}(i)\left(l_{a}+l_{a}^{2}\right) \alpha_{a}\right. \\
& \left.+\frac{1}{2 \pi}\left[\frac{d_{j k}^{i}}{2} \alpha_{Y_{i j k}}(\mu)\left(-\frac{\tilde{d}_{m n}^{p}}{2} q_{Y_{m n p}} \alpha_{Y_{m n p}}+2 c_{r} l_{a} \alpha_{a}\right)+2 C_{a}(i) b_{a} \alpha_{a}^{2}\right] \ln \left(\frac{\mathrm{GUT}}{\mu}\right)\right\} \\
& +\frac{M_{0} F_{\phi}}{2 \pi}\left[\frac{d_{j k}^{i}}{2} \alpha_{Y_{i j k}}\left(-q_{Y_{i k l}}+\frac{1}{2 \pi}\left[\frac{\tilde{d}_{m n}^{p}}{2} q_{Y_{m n p}} \alpha_{Y_{m n p}}-2 c_{r} l_{r} \alpha_{r}\right] \ln \left(\frac{M_{\mathrm{GUT}}}{\mu}\right)\right)+2 C_{a}(i) l_{a} \frac{\alpha_{a}^{2}}{\alpha_{a}(\mathrm{GUT})}\right] \\
& -\frac{F_{\phi}^{2}}{8 \pi}\left[\frac{d_{j k}^{i}}{2} \frac{1}{2 \pi}\left(\frac{\tilde{d}_{m n}^{p}}{2} \alpha_{Y_{m n p}}-2 c_{r} \alpha_{r}\right) \alpha_{Y_{i j k}}-2 C_{a}(i) \frac{b_{a}}{2 \pi} \alpha_{a}^{2}\right]-\left(1-n_{i}\right) M_{0}^{2},
\end{aligned}
$$

with $q_{Y_{i j k}} \equiv 3-\left(n_{i}+n_{j}+n_{k}\right)=3-a_{i j k}$. Again, we can check that for $q_{Y_{i j k}}=1$ or negligible Yukawa couplings, the soft scalar masses apparent unify at $\mu_{M i}$ defined above

$$
\begin{aligned}
- & m_{i}^{2}+\left(1-n_{i}\right) M_{0}^{2} \\
= & \pi \alpha M_{0}^{2}\left\{2 \frac{d_{j k}^{i}}{2} \alpha_{Y_{i j k}}(\mu)-4 C_{a}(i) \alpha_{a}+\frac{1}{2 \pi}\left[\frac{d_{j k}^{i}}{2} \alpha_{Y_{i j k}}\left(-\frac{\tilde{d}_{m n}^{p}}{2} \alpha_{Y_{m n p}}+2 c_{r} \alpha_{a}\right)+2 C_{a}(i) b_{a} \alpha_{a}^{2}\right] 2 \pi^{2} \alpha\right\} \\
& +2 \pi \alpha M_{0}^{2}\left[\frac{d_{j k}^{i}}{2} \alpha_{Y_{i j k}}\left(-1+\frac{1}{2 \pi}\left[\frac{\tilde{d}_{m n}^{p}}{2} \alpha_{Y_{m n p}}-2 c_{r}^{i} \alpha_{i}\right] 2 \pi^{2} \alpha\right)+2 C_{a}(i)\left(\alpha_{a}-\frac{1}{2 \pi} b_{a} \alpha_{a}^{2} 2 \pi^{2} \alpha\right)\right] \\
& -2 \pi^{3} \alpha^{2} M_{0}^{2}\left[\frac{d_{j k}^{i}}{2} \frac{1}{2 \pi}\left(\frac{\tilde{d}_{m n}^{p}}{2} \alpha_{Y_{m n p}}-2 c_{r}^{i} \alpha_{i}\right) \alpha_{Y_{i j k}}-2 C_{a}(i) \frac{b_{a}}{2 \pi} \alpha_{a}^{2}\right] \\
= & 0 .
\end{aligned}
$$

The subleading terms within $\partial^{2} Z_{i} / \partial T^{2}$ are crucial for the exact cancelation of anomaly mediation and RGE effects.

So the numerical results of 'mirage' unification can be proved rigourously with our analytical expressions.

\subsection{Deflection in mirage mediation from the Kahler potential}

It is well known that AMSB is bothered by tachyonic slepton problems. Such a problem in AMSB can be solved by the deflection of RGE trajectory with the introduction of messenger sector. There are two possible ways to deflect the AMSB trajectory with the presence of messengers, either by pseudo-moduli field [19, 20] or holomorphic terms (for messengers) in the Kahler potential [59]. Mirage mediation is a typical mixed modulus-anomaly mediation 
scenario. So the messenger sector, which can give additional gauge or Yukawa mediation contributions, can also be added in the Kahler potential.

The Kahler potential involving the vector-like messengers $\bar{P}_{i}, P_{i}$ contain the ordinary kinetic terms as well as new holomorphic terms

$$
K \supseteq \phi^{\dagger} \phi\left[Z_{P_{i}, \bar{P}_{i}}\left(T^{\dagger}, T\right)\left(P_{i}^{\dagger} P_{i}+\bar{P}_{i}^{\dagger} \bar{P}_{i}\right)+\left(\tilde{Z}_{P_{i}, \bar{P}_{i}}\left(T^{\dagger}, T\right) c_{P} \bar{P}_{i} P_{i}+\text { h.c. }\right)\right],
$$

with

$$
Z_{P_{i}, \bar{P}_{i}}\left(T^{\dagger}, T\right)=\frac{1}{\left(T+T^{\dagger}\right)^{n_{P}}}, \quad \tilde{Z}_{P_{i}, \bar{P}_{i}}\left(T^{\dagger}, T\right)=\frac{1}{\left(T+T^{\dagger}\right)^{\tilde{n}_{P}}}
$$

After normalizing and rescaling each superfield with the compensator field $\Phi \rightarrow \phi \Phi$ and substituting the F-term VEVs of the compensator field $\phi=1+F_{\phi} \theta^{2}$, the relevant Kahler potential reduces to

$$
W=\int d^{4} \theta \frac{\phi^{\dagger}}{\phi} \frac{1}{\left(T+T^{\dagger}\right)^{\tilde{n}_{P}-n_{P}}}\left(c_{P} R \bar{P} P\right) .
$$

For simply, we define $\tilde{n}_{P}-n_{P} \equiv a_{P}$. Especially, $a_{P}=n_{P}$ for $\tilde{n}_{P}=0$.

The SUSY breaking effects can be taken into account by introducing a spurion superfields $R$ with the spurion VEV as

$$
R \equiv M_{R}+\theta^{2} F_{R}=\frac{1}{(2 T)^{a_{P}}}\left(F_{\phi}-\frac{a_{P}}{2 T} F_{T}\right)+\theta^{2}\left[a_{P}\left(a_{P}+1\right) \frac{\left|F_{T}\right|^{2}}{4 T^{2}}-\left|F_{\phi}\right|^{2}\right],
$$

with the value of the deflection parameter

$$
d \equiv \frac{F_{R}}{M_{R} F_{\phi}}-1
$$

depending on the choice of $a_{P}$ and $\alpha$ which gives $d=-2$ for $a_{P}=0$. We can see that adding messenger sector in the Kahler potential within mirage mediation will display a new feature in contrast to the AMSB case which always predicts $d=-2$.

The appearance of spurion messenger threshold will affect the AMSB RGE trajectory after integrating out the heavy messenger modes. The soft SUSY breaking parameters can be obtained by substituting ' $d$ ' into the general formula given in the appendix. Note that we can derive the final results directly with its low energy analytical expressions. Besides, we can also add messenger-matter mixing to induce new Yukawa couplings between the messengers and the MSSM fields. In this case, new Yukawa mediated contributions will also contribute to the low energy soft SUSY parameters (See refs. [61-63] for an example in AMSB).

\subsection{Deflected mirage mediation with messenger-matter interactions}

In ordinary deflected mirage mediation SUSY breaking scenarios, additional messengers are introduced merely to amend the gauge beta functions which will subsequently feed into the low energy soft SUSY breaking parameters. In general, it is possible that the messengers will share some new Yukawa-type interactions with the visible (N)MSSM superfields, which 
subsequently will appear in the anomalous dimension of the superfields and contribute to the low energy soft SUSY breaking parameters. Such realizations have analogs in AMSB (see [61-63]) and can be readily extended to include the modulus mediation contributions.

Similar to the deflected mirage mediation scenarios, the superpotential include possible pseudo-modulus superfields $X$, the relevant nearly flat superpotential $W(X)$ to determine the deflection and a new part that includes messenger-matter interactions

$$
W_{m m}=\lambda_{\phi i j} X Q_{i} Q_{j}+y_{i j k} Q_{i} Q_{j} Q_{k}+W(X),
$$

with the Kahler potential

$$
K_{m}=Z_{U}\left(T+T^{\dagger}, \frac{\mu}{\sqrt{\phi^{\dagger} \phi}}\right) \frac{1}{\left(T+T^{\dagger}\right)^{n_{Q_{i}}}} Q_{i}^{\dagger} Q_{i} .
$$

Here ' $\phi^{\prime}$ denotes the compensator field with Weyl weight 1 . The indices ' $i, j^{\prime}$ run over all MSSM and messenger fields and the subscripts ' $U, D^{\prime}$ denote the case upon and below the messenger threshold, respectively.

After integrating out the heavy messenger fields, the visible sector superfields $Q_{a}$ will receive wavefunction normalization

$$
\mathcal{L}=\int d^{4} \theta Q_{a}^{\dagger} Z_{D}^{a b}\left(T+T^{\dagger}, \frac{\mu}{\sqrt{\phi^{\dagger} \phi}}, \sqrt{\frac{X^{\dagger} X}{\phi^{\dagger} \phi}}\right) Q_{b}+\int d^{2} \theta y_{a b c} Q^{a} Q^{b} Q^{c},
$$

which can give additional contributions to soft supersymmetry breaking parameters. Here the analytic continuing threshold superfield ' $X^{\prime}$ will trigger SUSY breaking mainly from the anomaly induced SUSY breaking effects with the form $\langle X\rangle=M+\theta^{2} F_{X}$. So we have

$$
\tilde{X} \equiv \frac{X}{\phi}=\frac{M+F_{X} \theta^{2}}{1+F_{\phi} \theta^{2}} \equiv M\left(1+d F_{\phi} \theta^{2}\right),
$$

with the value of the deflection parameter ' $d$ ' determined by the form of superpotential $W(X)$.

Integrating out the messengers, the messenger-matter interactions will cause the discontinuity of the anomalous dimension upon and below the threshold. Such discontinuity will appear not only directly in the expressions for the trilinear couplings but also indirectly in the soft scalar masses. For example, the trilinear couplings at the messenger scale receive additional contributions

$$
\begin{aligned}
\left.\Delta A_{i j k}\right|_{\mu=M} & =\left.\sum_{a=i, j, k} \frac{d}{2} F_{\phi} \frac{\partial}{\partial \ln |X|} \ln \left[e^{-K_{0} / 3} Z_{a}(\mu, X, T)\right]\right|_{\mu=M} \\
& =\left.\frac{d}{2} F_{\phi} \sum_{a=i, j, k} \Delta G_{i}\right|_{\mu=M} .
\end{aligned}
$$

We know that large trilinear couplings, especially $A_{t}$, is welcome in low energy phenomenological studies to reduce fine tuning and increase the Higgs mass. So the introduction of messenger-matter interactions can open new possibilities for mirage phenomenology. 


\section{Conclusions}

We derive explicitly the soft SUSY breaking parameters at arbitrary low energy scale in the (deflected) mirage type mediation scenarios with possible gauge or Yukawa mediation contributions. Based on the Wilsonian effective action after integrating out the messengers, we obtain analytically the boundary value (at the GUT scale) dependencies of the effective wavefunctions and gauge kinetic terms. Note that the messenger scale dependencies of the effective wavefunctions and gauge kinetic terms had already been discussed in GMSB. The RGE boundary value dependencies, which is a special feature in (deflected) mirage type mediation, is the key new ingredients in this study. The appearance of 'mirage' unification scale in mirage mediation is proved rigorously with our analytical results. We also discuss briefly the new features in deflected mirage mediation scenario in the case the deflection comes purely from the Kahler potential and the case with messenger-matter interactions.

We should note that our approach is in principle different from that of ref. [47] in which the soft SUSY breaking parameters are obtained by numerical RGE evolution, matching and threshold corrections. For example, mixed gauge-modulus mediation contributions, which will not appear in previous approach, will be necessarily present for the soft scalar masses in our approach.

\section{Acknowledgments}

This work was supported by the Natural Science Foundation of China under grant numbers 11675147,11775012; by the Innovation Talent project of Henan Province under grant number 15HASTIT017; by the Young Core Instructor Foundation of Henan Education Department.

\section{A Coefficients in wavefunction expansion}

We can construct the RGE invariants

$$
\frac{d}{d t} \ln Z_{i}=\sum_{l=y_{t}, y_{b}, y_{\tau}} A_{l} \frac{d \ln y_{l}}{d t}+\sum_{l=g_{3}, g_{2}, g_{1}} B_{l} \frac{d \ln g_{l}}{d t},
$$

by solving the equation in the basis of $\left(y_{t}^{2}, y_{b}^{2}, y_{\tau}^{2}, g_{3}^{2}, g_{2}^{2}, g_{1}^{2}\right)$

$$
\left(\begin{array}{cccccc}
6 & 1 & 0 & 0 & 0 & 0 \\
1 & 6 & 3 & 0 & 0 & 0 \\
0 & 1 & 4 & 0 & 0 & 0 \\
-\frac{16}{3} & -\frac{16}{3} & 0 & b_{3} & 0 & 0 \\
-3 & -3 & -3 & 0 & b_{2} & 0 \\
-\frac{13}{15} & -\frac{7}{15} & -\frac{9}{5} & 0 & 0 & b_{1}
\end{array}\right)\left(\begin{array}{l}
A_{t} \\
A_{b} \\
A_{\tau} \\
B_{3} \\
B_{2} \\
B_{1}
\end{array}\right)=\left(\begin{array}{l}
-2 c_{1} \\
-2 c_{2} \\
-2 c_{3} \\
-2 d_{1} \\
-2 d_{2} \\
-2 d_{3}
\end{array}\right),
$$

with $c_{1}, c_{2}, c_{3}, d_{1}, d_{2}, d_{3}$ the relevant coefficients of $\left(y_{t}^{2}, y_{b}^{2}, y_{\tau}^{2}, g_{3}^{2}, g_{2}^{2}, g_{1}^{2}\right)$ within the anomalous dimension. So from

$$
\frac{d}{d t}\left[Z_{i}(\mu) \prod_{l=y_{t}, y_{b}, y_{\tau}}\left[y_{l}(\mu)\right]^{-A_{l}} \prod_{k=1,2,3}\left[g_{k}(\mu)\right]^{-B_{k}}\right]=0,
$$




\begin{tabular}{|c|c|c|c|c|c|c|}
\hline & $A_{1}\left(y_{t}\right)$ & $A_{2}\left(y_{b}\right)$ & $A_{3}\left(y_{\tau}\right)$ & $B_{3}\left(g_{3}\right)$ & $B_{2}\left(g_{2}\right)$ & $B_{1}\left(g_{1}\right)$ \\
\hline$Q_{3}$ & $-\frac{17}{61}$ & $-\frac{20}{61}$ & $\frac{5}{61}$ & $-\frac{128}{183}$ & $\frac{87}{61}$ & $-\frac{5}{183}$ \\
\hline$U_{3}$ & $-\frac{42}{61}$ & $\frac{8}{61}$ & $-\frac{2}{61}$ & $-\frac{48}{61}$ & $-\frac{108}{61}$ & $\frac{48}{671}$ \\
\hline$D_{3}$ & $\frac{8}{61}$ & $-\frac{48}{61}$ & $\frac{12}{61}$ & $-\frac{112}{183}$ & $-\frac{84}{61}$ & $\frac{112}{2013}$ \\
\hline$L_{3}$ & $-\frac{3}{61}$ & $\frac{18}{61}$ & $-\frac{35}{61}$ & $-\frac{80}{183}$ & $\frac{123}{61}$ & $-\frac{103}{2013}$ \\
\hline$E_{3}$ & $-\frac{6}{61}$ & $\frac{36}{61}$ & $-\frac{70}{61}$ & $-\frac{160}{183}$ & $-\frac{120}{61}$ & $\frac{160}{2013}$ \\
\hline$H_{u}$ & $-\frac{63}{61}$ & $\frac{12}{61}$ & $-\frac{3}{61}$ & $\frac{272}{183}$ & $\frac{21}{61}$ & $-\frac{89}{2013}$ \\
\hline$H_{d}$ & $\frac{9}{61}$ & $-\frac{54}{61}$ & $-\frac{17}{61}$ & $\frac{80}{61}$ & $-\frac{3}{61}$ & $-\frac{19}{671}$ \\
\hline$Q_{2}$ & 0 & 0 & 0 & $-\frac{16}{9}$ & 3 & $\frac{1}{99}$ \\
\hline$U_{2}$ & 0 & 0 & 0 & $-\frac{16}{9}$ & 0 & $\frac{16}{99}$ \\
\hline$D_{2}$ & 0 & 0 & 0 & $-\frac{16}{9}$ & 0 & $\frac{4}{99}$ \\
\hline$L_{2}$ & 0 & 0 & 0 & 0 & 3 & $\frac{1}{11}$ \\
\hline$E_{2}$ & 0 & 0 & 0 & 0 & 0 & $\frac{4}{11}$ \\
\hline
\end{tabular}

Table 1. Relevant coefficients in wavefunction expansion with $N_{F}=0$ messengers.

we have

$$
Z_{i}(\mu)=Z_{i}(\Lambda) \prod_{l=y_{t}, y_{b}, y_{\tau}}\left(\frac{y_{l}(\mu)}{y_{l}(\Lambda)}\right)^{A_{l}} \prod_{k=1,2,3}\left(\frac{g_{k}(\mu)}{g_{k}(\Lambda)}\right)^{B_{k}}
$$

The general expressions of wavefunction at ordinary scale $\mu$ below the messenger scale $M$ are given as

$$
\begin{gathered}
Z_{i}(\mu)=Z_{i}(\Lambda) \prod_{l=y_{t}, y_{b}, y_{\tau}}\left(\frac{y_{l}(M)}{y_{l}(\Lambda)}\right)^{A_{l}} \prod_{k=1,2,3}\left(\frac{g_{k}(M)}{g_{k}(\Lambda)}\right)^{B_{k}} \prod_{k=y_{U}}\left(\frac{y_{k}(M)}{y_{k}(\Lambda)}\right)^{C_{k}} \\
\prod_{l=y_{t}, y_{b}, y_{\tau}}\left(\frac{y_{l}(\mu)}{y_{l}(M)}\right)^{A_{l}^{\prime}} \prod_{k=1,2,3}\left(\frac{g_{k}(\mu)}{g_{k}(M)}\right)^{B_{k}^{\prime}},
\end{gathered}
$$

with $y_{U}$ the interactions involving the messengers which will be integrated below the messenger scale. The coefficients are listed in table 1 and table 2.

\section{B Low energy spectrum in deflected mirage mediation}

In order to show some essential features of our effective theory results, we list the predicted soft SUSY breaking parameters in deflected mirage mediation mechanism with $N_{F}$ messengers in $\mathbf{5} \oplus \overline{\mathbf{5}}$ representations of $\mathrm{SU}(5)$.

At energy $\mu$ below the messenger thresholds, we have

- The gaugino masses:

$$
M_{i}(\mu)=l_{a} M_{0} \frac{g_{i}^{2}(\mu)}{g_{a}^{2}(\mathrm{GUT})}+\frac{F_{\phi}}{16 \pi^{2}} b_{i} g_{i}^{2}(\mu)-d \frac{F_{\phi}}{16 \pi^{2}} N_{F} g_{i}^{2}(\mu),
$$




\begin{tabular}{|l|c|c|c|}
\hline & $B_{g_{3}}(i)$ & $B_{g_{2}}(i)$ & $B_{g_{1}}(i)$ \\
\hline$Q_{3}$ & $\left(-\frac{128}{183},-\frac{64}{61},-\frac{128}{61}, \frac{128}{61}\right)$ & $\left(\frac{87}{61}, \frac{87}{122}, \frac{29}{61}, \frac{87}{305}\right)$ & $\left(-\frac{5}{183},-\frac{55}{2318},-\frac{55}{2623},-\frac{55}{3233}\right)$ \\
\hline$U_{3}$ & $\left(-\frac{48}{61},-\frac{72}{61},-\frac{144}{61}, \frac{144}{61}\right)$ & $\left(-\frac{108}{61},-\frac{54}{61},-\frac{36}{61},-\frac{108}{305}\right)$ & $\left(\frac{48}{671}, \frac{72}{1159}, \frac{144}{2623}, \frac{144}{3233}\right)$ \\
\hline$D_{3}$ & $\left(-\frac{112}{183},-\frac{56}{61},-\frac{112}{61}, \frac{112}{61}\right)$ & $\left(-\frac{84}{61},-\frac{42}{61},-\frac{28}{61},-\frac{84}{305}\right)$ & $\left(\frac{112}{2013}, \frac{56}{1159}, \frac{112}{2623}, \frac{112}{3233}\right)$ \\
\hline$L_{3}$ & $\left(-\frac{80}{183},-\frac{40}{61},-\frac{80}{61}, \frac{80}{61}\right)$ & $\left(\frac{123}{61}, \frac{123}{122}, \frac{41}{61}, \frac{123}{305}\right)$ & $\left(-\frac{103}{2013},-\frac{103}{2318},-\frac{103}{2623},-\frac{103}{3233}\right)$ \\
\hline$E_{3}$ & $\left(-\frac{160}{183},-\frac{80}{61},-\frac{160}{61}, \frac{160}{61}\right)$ & $\left(-\frac{120}{61},-\frac{60}{61},-\frac{40}{61},-\frac{24}{61}\right)$ & $\left(\frac{160}{2013}, \frac{80}{1159}, \frac{160}{2623}, \frac{160}{3233}\right)$ \\
\hline$H_{u}$ & $\left(\frac{272}{183}, \frac{136}{61}, \frac{272}{61},-\frac{272}{61}\right)$ & $\left(\frac{21}{61}, \frac{21}{122}, \frac{7}{61}, \frac{21}{305}\right)$ & $\left(-\frac{89}{2013},-\frac{89}{2318},-\frac{89}{2623},-\frac{89}{3233}\right)$ \\
\hline$H_{d}$ & $\left(\frac{80}{61}, \frac{120}{61}, \frac{240}{61},-\frac{240}{61}\right)$ & $\left(-\frac{3}{61},-\frac{3}{122},-\frac{1}{61},-\frac{3}{305}\right)$ & $\left(-\frac{19}{671},-\frac{3}{122},-\frac{57}{2623},-\frac{57}{3233}\right)$ \\
\hline$Q_{2}$ & $\left(-\frac{16}{9},-\frac{8}{3},-\frac{16}{3}, \frac{16}{3}\right)$ & $\left(3, \frac{3}{2}, 1, \frac{3}{5}\right)$ & $\left(\frac{1}{99}, \frac{1}{144}, \frac{1}{129}, \frac{1}{159}\right)$ \\
\hline$U_{2}$ & $\left(-\frac{16}{9},-\frac{8}{3},-\frac{16}{3}, \frac{16}{3}\right)$ & $(0,0,0,0)$ & $\left(\frac{16}{99}, \frac{8}{57}, \frac{16}{129}, \frac{16}{159}\right)$ \\
\hline$D_{2}$ & $\left(-\frac{16}{9},-\frac{8}{3},-\frac{16}{3}, \frac{16}{3}\right)$ & $(0,0,0,0)$ & $\left(\frac{4}{99}, \frac{2}{57}, \frac{4}{129}, \frac{4}{159}\right)$ \\
\hline$L_{2}$ & $(0,0,0,0)$ & $\left(3, \frac{3}{2}, 1, \frac{3}{5}\right)$ & $\left(\frac{1}{11}, \frac{3}{38}, \frac{3}{43}, \frac{3}{53}\right)$ \\
\hline$E_{2}$ & $(0,0,0,0)$ & $(0,0,0,0)$ & $\left(\frac{4}{11}, \frac{6}{19}, \frac{12}{43}, \frac{12}{53}\right)$ \\
\hline
\end{tabular}

Table 2. The coefficients with $N_{F}=0,1,2,4$ messengers without new Yukawa couplings involving the messengers-matter interactions. The coefficients for $y_{t}, y_{b}, y_{\tau}$, namely $A_{1}\left(y_{t}\right), A_{2}\left(y_{b}\right), A_{3}\left(y_{\tau}\right)$, are the same as the case $N_{F}=1$.

with

$$
\left(b_{3}, b_{2}, b_{1}\right)=\left(-3,1, \frac{33}{5}\right)
$$

- The trilinear couplings $A_{t}, A_{b}$ and $A_{\tau}$.

Note that at the messenger scale, the third contribution $\partial_{X} Z_{i}$ vanishes. The trilinear $A_{t}$ term is given at arbitrary low energy scale $\mu<M$

$$
\begin{aligned}
& A_{t}(\mu)-q_{y_{t}} M_{0} \\
& =\frac{M_{0}}{2 \pi}\left[6 \frac{q_{y_{t}}}{2} \alpha_{y_{t}}(\mu)+\frac{q_{y_{b}}}{2} \alpha_{y_{b}}(\mu)-\frac{16}{3} l_{3} \alpha_{3}(\mu)-3 l_{2} \alpha_{2}(\mu)-\frac{13}{15} l_{1} \alpha_{1}(\mu)\right] \ln \left(\frac{M_{\mathrm{GUT}}}{\mu}\right) \\
& \quad+\frac{F_{\phi}}{4 \pi}\left[6 \alpha_{y_{t}}(\mu)+\alpha_{y_{b}}(\mu)-\frac{16}{3} \alpha_{3}(\mu)-3 \alpha_{2}(\mu)-\frac{13}{15} \alpha_{1}(\mu)\right]+\delta_{G} .
\end{aligned}
$$

Note that additional GMSB-type contributions are

$$
\begin{aligned}
\delta_{G}= & d \frac{F_{\phi}}{8 \pi} \sum_{k=1,2,3} \sum_{F=Q_{L}^{3}, U_{3}, H_{u}}\left[\left(B_{k}(F)-B_{k}^{\prime}(F)\right)\left(b_{k}+N_{F}\right) \alpha_{k}(M)+B_{k}^{\prime}(F) N_{F} \alpha_{k}(\mu)\right] \\
& +d \frac{F_{\phi}}{8 \pi} \sum_{y_{l}=y_{t}, y_{b}, y_{\tau}} \sum_{k=1,2,3} A_{l}^{\prime} \frac{1}{4 \pi^{2}} N_{F} \tilde{c}_{r}\left(y_{l}\right) \alpha_{r}^{2}(\mu) \ln \left(\frac{M}{\mu}\right) \\
= & d \frac{F_{\phi}}{8 \pi}\left[-2 \frac{1}{8 \pi^{2}} N_{F}\left(\frac{16}{3} \alpha_{3}^{2}(\mu)+3 \alpha_{2}^{2}(\mu)+\frac{13}{15} \alpha_{1}^{2}(\mu)\right) \ln \left(\frac{M}{\mu}\right)\right]
\end{aligned}
$$


with $2 \tilde{c}_{r}\left(y_{l}\right)$ the coefficients of $g_{r}^{2}$ within $-16 \pi^{2} \beta_{y_{l}}$ and

$$
\sum_{F=Q_{L}^{3}, U_{3}, H_{u}} B_{k}(F)=\sum_{F=Q_{L}^{3}, U_{3}, H_{u}} B_{k}^{\prime}(F)=0 .
$$

The trilinear $A_{b}$ term is

$$
\begin{aligned}
& A_{b}(\mu)-q_{y_{b}} M_{0} \\
& =\frac{M_{0}}{2 \pi}\left[\frac{q_{y_{t}}}{2} \alpha_{y_{t}}(\mu)+6 \frac{q_{y_{b}}}{2} \alpha_{y_{b}}(\mu)+\frac{q_{y_{\tau}}}{2} \alpha_{y_{\tau}}(\mu)-\frac{16}{3} l_{3} \alpha_{3}(\mu)-3 l_{2} \alpha_{2}(\mu)-\frac{7}{15} l_{1} \alpha_{1}(\mu)\right] \ln \left(\frac{M_{\mathrm{GUT}}}{\mu}\right) \\
& \quad+\frac{F_{\phi}}{4 \pi}\left[\alpha_{y_{t}}(\mu)+6 \alpha_{y_{b}}(\mu)+\alpha_{y_{\tau}}(\mu)-\frac{16}{3} \alpha_{3}(\mu)-3 \alpha_{2}(\mu)-\frac{7}{15} \alpha_{1}(\mu)\right] \\
& \quad+d \frac{F_{\phi}}{8 \pi}\left[-2 \frac{1}{8 \pi^{2}} N_{F}\left(\frac{16}{3} \alpha_{3}^{2}(\mu)+3 \alpha_{2}^{2}(\mu)+\frac{7}{15} \alpha_{1}^{2}(\mu)\right) \ln \left(\frac{M}{\mu}\right)\right] .
\end{aligned}
$$

The trilinear $A_{\tau}$ term is

$$
\begin{aligned}
& A_{\tau}(\mu)-q_{y_{\tau}} M_{0} \\
& =\frac{M_{0}}{2 \pi}\left[3 \frac{q_{y_{b}}}{2} \alpha_{y_{b}}(\mu)+4 \frac{q_{y_{\tau}}}{2} \alpha_{y_{\tau}}(\mu)-3 l_{2} \alpha_{2}(\mu)-\frac{9}{5} l_{1} \alpha_{1}(\mu)\right] \ln \left(\frac{M_{\mathrm{GUT}}}{\mu}\right) \\
& \quad+\frac{F_{\phi}}{4 \pi}\left[3 \alpha_{y_{b}}(\mu)+4 \alpha_{y_{\tau}}(\mu)-3 \alpha_{2}(\mu)-\frac{9}{5} \alpha_{1}(\mu)\right] \\
& \quad+d \frac{F_{\phi}}{8 \pi}\left[-2 \frac{1}{8 \pi^{2}} N_{F}\left(3 \alpha_{2}^{2}(\mu)+\frac{9}{5} \alpha_{1}^{2}(\mu)\right) \ln \left(\frac{M}{\mu}\right)\right] .
\end{aligned}
$$

- The soft SUSY breaking scalar masses are parameterized by several terms:

$$
-m_{0}^{2}=-\left(1-n_{i}\right) M_{0}^{2}+\delta_{I}+\delta_{I I}+\delta_{I I I}+\delta_{I V}+\delta_{V}
$$

The anomalous dimension of $Z_{i}$ is supposed to take the form

$$
G^{i} \equiv \frac{d \ln Z_{i}}{d \ln \mu}=-\frac{1}{2 \pi}\left(\frac{1}{2} d_{k l}^{i} \alpha_{\lambda_{i k l}}-2 C_{a}(i) \alpha_{a}\right)
$$

with $\alpha_{\lambda_{i k l}}=\lambda_{i k l}^{2} / 4 \pi$ and $\alpha_{a}=g_{a}^{2} / 4 \pi$.

- Pure modulus mediation contributions

$$
\begin{aligned}
\delta_{I}= & \frac{M_{0}^{2}}{2 \pi} \ln \left(\frac{M_{\mathrm{GUT}}}{\mu}\right)\left\{\frac{d_{j k}^{i}}{2}\left(q_{Y_{i j k}}^{2}+q_{Y_{i j k}}\right) \alpha_{Y_{i j k}}(\mu)-2 C_{a}(i)\left(l_{a}+l_{a}^{2}\right) \alpha_{a}\right. \\
& \left.+\frac{1}{2 \pi}\left[\frac{d_{j k}^{i}}{2} \alpha_{Y_{i j k}}(\mu)\left(-\frac{\tilde{d}_{m n}^{p}}{2} q_{Y_{m n p}} \alpha_{Y_{m n p}}+2 c_{r} l_{a} \alpha_{a}\right)+2 C_{a}(i) b_{a} \alpha_{a}^{2}\right] \ln \left(\frac{\mathrm{GUT}}{\mu}\right)\right\} .
\end{aligned}
$$


- Pure anomaly mediation contributions

$$
\begin{aligned}
\delta_{I I} & =\frac{F_{\phi}^{2}}{4} \frac{\partial^{2}}{\partial(\ln \mu)^{2}} \ln \left[e^{-K_{0} / 3} Z_{i}\right] \\
& =-\frac{F_{\phi}^{2}}{8 \pi} \frac{\partial}{\partial(\ln \mu)}\left[\frac{1}{2} d_{k l}^{i} \alpha_{\lambda_{i k l}}-2 C_{a}(i) \alpha_{a}\right], \\
& =-\frac{F_{\phi}^{2}}{8 \pi}\left[\frac{1}{2} d_{k l}^{i} \alpha_{\lambda_{i k l}} 2 G_{\lambda_{i k l}}^{-}-2 C_{a}(i) \frac{1}{2 \pi} b_{a} \alpha_{a}^{2}\right],
\end{aligned}
$$

with the beta function for Yukawa coupling $\lambda_{i k l}$ being

$$
\frac{d \ln \lambda_{i k l}}{d \ln \mu}=G_{\lambda_{i k l}}=\frac{1}{4 \pi}\left[\frac{1}{2} d_{m n}^{p} \alpha_{\lambda_{m n p}}-2 c_{r} \alpha_{r}\right] .
$$

- Pure gauge mediation contributions

As no new interactions involving the messengers are present, we have

$$
\begin{aligned}
\frac{\partial}{\partial \ln M} \ln \left[e^{-K_{0} / 3} Z_{i}\right]= & \frac{1}{4 \pi} \sum_{g_{k}}\left[\left(B_{k}-B_{k}^{\prime}\right)\left(b_{k}+N_{F}\right) \alpha_{k}(M)+B_{k}^{\prime} N_{F} \alpha_{k}(\mu)\right] \\
& +\sum_{Y_{l}} A_{l}^{\prime} \frac{\tilde{c}_{r}}{4 \pi^{2}} \Delta b_{r} \alpha_{r}^{2}(\mu) \ln \left(\frac{M}{\mu}\right)
\end{aligned}
$$

So

$$
\begin{aligned}
\delta_{I I I} & =d^{2} \frac{F_{\phi}^{2}}{4} \frac{\partial^{2}}{\partial(\ln M)^{2}} \ln \left[e^{-K_{0} / 3} Z_{i}\right] \\
& =d^{2} \frac{F_{\phi}^{2}}{32 \pi^{2}}\left\{\sum_{g_{k}}\left[\left(B_{k}-B_{k}^{\prime}\right)\left(b_{k}+N_{F}\right)^{2} \alpha_{k}^{2}(M)+B_{k}^{\prime} N_{F}^{2} \alpha^{2}(\mu)\right]+\sum_{Y_{l}} A_{l}^{\prime} \frac{\tilde{c}_{r}}{4 \pi^{2}} \Delta b_{r} \alpha_{r}^{2}(\mu)\right\} .
\end{aligned}
$$

Here

$$
\begin{aligned}
\frac{\partial}{\partial \ln M} \alpha_{k}(M) & =\frac{b_{k}^{+}}{2 \pi} \alpha_{k}(M), \\
\frac{\partial}{\partial \ln M} \alpha_{k}(\mu, M) & =\frac{b_{k}^{+}-b_{k}^{-}}{2 \pi} \alpha_{k}(\mu, M) \equiv \frac{\Delta b_{k}}{2 \pi} \alpha_{k}(\mu, M) .
\end{aligned}
$$

- The gauge-anomaly interference term

$$
\begin{aligned}
\delta_{I V}= & -\frac{d F_{\phi}^{2}}{2} \frac{\partial^{2}}{\partial \ln M \partial \ln \mu} \ln \left[e^{-K_{0} / 3} Z_{i}(\mu, X, T)\right] \\
= & -\frac{d F_{\phi}^{2}}{2} \frac{\partial}{\partial \ln \mu}\left\{\frac{1}{4 \pi} \sum_{g_{k}}\left[\left(B_{k}-B_{k}^{\prime}\right)\left(b_{k}+N_{F}\right) \alpha_{k}(M)+B_{k}^{\prime} N_{F} \alpha_{k}(\mu)\right]\right. \\
& \left.+\sum_{Y_{l}} A_{l}^{\prime} \frac{\tilde{c}_{r}}{4 \pi^{2}} \Delta b_{r} \alpha_{r}^{2}(\mu) \ln \left(\frac{M}{\mu}\right)\right\} \\
= & -\frac{d F_{\phi}^{2}}{16 \pi^{2}} B_{k}^{\prime} b_{k} N_{F} \alpha_{k}^{2}(\mu)-d F_{\phi}^{2} \frac{\tilde{c}_{r}}{8 \pi^{2}} A_{l}^{\prime} \Delta b_{r} \alpha_{r}^{2}(\mu) .
\end{aligned}
$$


- The modulus-anomaly and modulus-gauge interference terms are given as

$$
\begin{aligned}
\delta_{V}= & -\frac{F_{T} F_{\phi}}{2} \frac{\partial^{2}}{\partial T \partial \ln \mu} \ln \left[e^{-K_{0} / 3} Z_{i}\right]+\frac{d F_{T} F_{\phi}}{2} \frac{\partial^{2}}{\partial T \partial \ln |X|} \ln \left[e^{-K_{0} / 3} Z_{i}\right] \\
= & \frac{F_{T} F_{\phi}}{4 \pi} \frac{\partial}{\partial T}\left[\frac{1}{2} d_{k l}^{i} \alpha_{\lambda_{i k l}}-2 C_{a}(i) \alpha_{a}\right] \\
& +\frac{d F_{T} F_{\phi}}{2} \frac{\partial}{\partial T}\left\{\frac{1}{4 \pi} \sum_{g_{k}}\left[\left(B_{k}-B_{k}^{\prime}\right)\left(b_{k}+N_{F}\right) \alpha_{k}(M)+B_{k}^{\prime} N_{F} \alpha_{k}(\mu)\right]\right. \\
& \left.+\sum_{Y_{l}} A_{l}^{\prime} \frac{\tilde{c}_{r}}{4 \pi^{2}} \Delta b_{r} \alpha_{r}^{2}(\mu) \ln \left(\frac{M}{\mu}\right)\right\} \\
= & \frac{M_{0} F_{\phi}}{2 \pi}\left[\frac{d_{k l}^{i}}{2} \alpha_{\lambda_{i k l}}\left(-q_{y_{\lambda_{i k l}}}+\frac{1}{2 \pi}\left[\frac{d_{m n}^{p}}{2} q_{y_{\lambda_{m n}}} \alpha_{\lambda_{m n p}}-2 c_{r} l_{r} \alpha_{r}\right] \ln \left[\frac{M_{G}}{\mu}\right]\right)\right. \\
& \left.+2 C_{a}(i) \frac{l_{a}}{T} \frac{\alpha_{a}^{2}}{\alpha_{a}(\mathrm{GUT})}\right] \\
& -\frac{d F_{\phi} M_{0}}{4 \pi} \sum_{g_{k}}\left[\left(B_{k}-B_{k}^{\prime}\right)\left(b_{k}+N_{F}\right) l_{k} \frac{\alpha_{k}^{2}(M)}{\alpha_{k}(\mathrm{GUT})}+B_{k}^{\prime} N_{F} l_{k} \frac{\alpha_{k}^{2}(\mu)}{\alpha_{k}(\mathrm{GUT})}\right] \\
& \left.-\sum_{Y_{l}} A_{l}^{\prime} \frac{d M_{0} F_{\phi}}{4 \pi^{2}} \tilde{c}_{r} \Delta b_{r} l_{r} \frac{2 \alpha_{r}^{3}(\mu)}{\alpha_{r}(\mathrm{GUT})} \ln \left(\frac{M}{\mu}\right)\right\},
\end{aligned}
$$

with

$$
\frac{\partial}{\partial T} \alpha_{k}(\mu)=-\frac{l_{k}}{T} \frac{\alpha_{k}(\mu)}{\alpha_{k}(\mathrm{GUT})} \alpha_{k}(\mu) .
$$

Open Access. This article is distributed under the terms of the Creative Commons Attribution License (CC-BY 4.0), which permits any use, distribution and reproduction in any medium, provided the original author(s) and source are credited.

\section{References}

[1] ATLAS collaboration, Combined search for the Standard Model Higgs boson using up to $4.9 \mathrm{fb}^{-1}$ of pp collision data at $\sqrt{\mathrm{s}}=7 \mathrm{TeV}$ with the ATLAS detector at the LHC, Phys. Lett. B 710 (2012) 49 [arXiv:1202.1408] [INSPIRE].

[2] CMS collaboration, Combined results of searches for the standard model Higgs boson in pp collisions at $\sqrt{s}=7 \mathrm{TeV}$, Phys. Lett. B 710 (2012) 26 [arXiv:1202.1488] [INSPIRE].

[3] S. Kachru, R. Kallosh, A.D. Linde and S.P. Trivedi, de Sitter vacua in string theory, Phys. Rev. D 68 (2003) 046005 [hep-th/0301240] [INSPIRE].

[4] C.P. Burgess, R. Kallosh and F. Quevedo, de Sitter string vacua from supersymmetric D terms, JHEP 10 (2003) 056 [hep-th/0309187] [INSPIRE].

[5] A. Saltman and E. Silverstein, The Scaling of the no scale potential and de Sitter model building, JHEP 11 (2004) 066 [hep-th/0402135] [INSPIRE]. 
[6] J.P. Conlon, F. Quevedo and K. Suruliz, Large-volume flux compactifications: Moduli spectrum and D3/D7 soft supersymmetry breaking, JHEP 08 (2005) 007 [hep-th/0505076] [INSPIRE].

[7] K. Choi, A. Falkowski, H.P. Nilles, M. Olechowski and S. Pokorski, Stability of flux compactifications and the pattern of supersymmetry breaking, JHEP 11 (2004) 076 [hep-th/0411066] [INSPIRE].

[8] K. Choi, A. Falkowski, H.P. Nilles and M. Olechowski, Soft supersymmetry breaking in KKLT flux compactification, Nucl. Phys. B 718 (2005) 113 [hep-th/0503216] [INSPIRE].

[9] L. Randall and R. Sundrum, Out of this world supersymmetry breaking, Nucl. Phys. B 557 (1999) 79 [hep-th/9810155] [INSPIRE].

[10] G.F. Giudice, M.A. Luty, H. Murayama and R. Rattazzi, Gaugino mass without singlets, JHEP 12 (1998) 027 [hep-ph/9810442] [INSPIRE].

[11] K. Choi, K.S. Jeong and K.-i. Okumura, Phenomenology of mixed modulus-anomaly mediation in fluxed string compactifications and brane models, JHEP 09 (2005) 039 [hep-ph/0504037] [INSPIRE].

[12] I. Jack and D.R.T. Jones, Fayet-Iliopoulos D terms and anomaly mediated supersymmetry breaking, Phys. Lett. B 482 (2000) 167 [hep-ph/0003081] [INSPIRE].

[13] E. Katz, Y. Shadmi and Y. Shirman, Heavy thresholds, slepton masses and the mu term in anomaly mediated supersymmetry breaking, JHEP 08 (1999) 015 [hep-ph/9906296] [INSPIRE].

[14] N. Arkani-Hamed, D.E. Kaplan, H. Murayama and Y. Nomura, Viable ultraviolet insensitive supersymmetry breaking, JHEP 02 (2001) 041 [hep-ph/0012103] [INSPIRE].

[15] R. Sundrum, 'Gaugomaly' mediated SUSY breaking and conformal sequestering, Phys. Rev. D 71 (2005) 085003 [hep-th/0406012] [INSPIRE].

[16] K. Hsieh and M.A. Luty, Mixed gauge and anomaly mediation from new physics at 10-TeV, JHEP 06 (2007) 062 [hep-ph/0604256] [INSPIRE].

[17] Y. Cai and M.A. Luty, Minimal Gaugomaly Mediation, JHEP 12 (2010) 037 [arXiv: 1008.2024] [INSPIRE].

[18] T. Kobayashi, Y. Nakai and M. Sakai, (Extra)Ordinary Gauge/Anomaly Mediation, JHEP 06 (2011) 039 [arXiv: 1103.4912] [INSPIRE].

[19] A. Pomarol and R. Rattazzi, Sparticle masses from the superconformal anomaly, JHEP 05 (1999) 013 [hep-ph/9903448] [INSPIRE].

[20] R. Rattazzi, A. Strumia and J.D. Wells, Phenomenology of deflected anomaly mediation, Nucl. Phys. B 576 (2000) 3 [hep-ph/9912390] [INSPIRE].

[21] N. Okada, Positively deflected anomaly mediation, Phys. Rev. D 65 (2002) 115009 [hep-ph/0202219] [INSPIRE].

[22] N. Okada and H.M. Tran, Positively deflected anomaly mediation in the light of the Higgs boson discovery, Phys. Rev. D 87 (2013) 035024 [arXiv:1212.1866] [INSPIRE].

[23] F. Wang, W. Wang, J.M. Yang and Y. Zhang, Heavy colored SUSY partners from deflected anomaly mediation, JHEP 07 (2015) 138 [arXiv:1505.02785] [INSPIRE]. 
[24] F. Wang, J.M. Yang and Y. Zhang, Radiative natural SUSY spectrum from deflected AMSB scenario with messenger-matter interactions, JHEP 04 (2016) 177 [arXiv:1602.01699] [INSPIRE].

[25] F. Wang, L. Wu, J.M. Yang and M. Zhang, 750 GeV diphoton resonance, 125 GeV Higgs and muon $g-2$ anomaly in deflected anomaly mediation SUSY breaking scenarios, Phys. Lett. B 759 (2016) 191 [arXiv:1512.06715] [INSPIRE].

[26] L.L. Everett, I.-W. Kim, P. Ouyang and K.M. Zurek, Deflected Mirage Mediation: A Framework for Generalized Supersymmetry Breaking, Phys. Rev. Lett. 101 (2008) 101803 [arXiv: 0804.0592] [INSPIRE].

[27] L.L. Everett, I.-W. Kim, P. Ouyang and K.M. Zurek, Moduli Stabilization and Supersymmetry Breaking in Deflected Mirage Mediation, JHEP 08 (2008) 102 [arXiv:0806.2330] [INSPIRE].

[28] L.L. Everett, T. Garon, B.L. Kaufman and B.D. Nelson, Mirage Models Confront the LHC: III. Deflected Mirage Mediation, Phys. Rev. D 93 (2016) 055031 [arXiv:1510.05692] [INSPIRE].

[29] B. Altunkaynak, B.D. Nelson, L.L. Everett, I.-W. Kim and Y. Rao, Phenomenological Implications of Deflected Mirage Mediation: Comparison with Mirage Mediation, JHEP 05 (2010) 054 [arXiv: 1001.5261] [INSPIRE].

[30] B. Kaufman and B.D. Nelson, Mirage Models Confront the LHC: II. Flux-Stabilized Type IIB String Theory, Phys. Rev. D 89 (2014) 085029 [arXiv:1312.6621] [INSPIRE].

[31] H. Abe and J. Kawamura, The $126 \mathrm{GeV}$ Higgs boson mass and naturalness in (deflected) mirage mediation, JHEP 07 (2014) 077 [arXiv: 1405.0779] [INSPIRE].

[32] L.L. Everett, T. Garon, B.L. Kaufman and B.D. Nelson, Mirage Models Confront the LHC: III. Deflected Mirage Mediation, Phys. Rev. D 93 (2016) 055031 [arXiv:1510.05692] [INSPIRE].

[33] K. Huitu, P.N. Pandita and P. Tiitola, Renormalization group invariants and sum rules in the deflected mirage mediation supersymmetry breaking, Phys. Rev. D 92 (2015) 075037 [arXiv: 1505.03455] [INSPIRE].

[34] V. Barger, L.L. Everett and T.S. Garon, Electroweak Naturalness and Deflected Mirage Mediation, Phys. Rev. D 93 (2016) 075024 [arXiv:1512.05011] [INSPIRE].

[35] H. Baer, V. Barger, H. Serce and X. Tata, Natural generalized mirage mediation, Phys. Rev. D 94 (2016) 115017 [arXiv: 1610.06205] [INSPIRE].

[36] T. Kobayashi, H. Makino, K.-i. Okumura, T. Shimomura and T. Takahashi, TeV scale mirage mediation in NMSSM, JHEP 01 (2013) 081 [arXiv:1204.3561] [INSPIRE].

[37] K. Hagimoto, T. Kobayashi, H. Makino, K.-i. Okumura and T. Shimomura, Phenomenology of NMSSM in TeV scale mirage mediation, JHEP 02 (2016) 089 [arXiv:1509.05327] [INSPIRE].

[38] A. Pierce and J. Thaler, Prospects for Mirage Mediation, JHEP 09 (2006) 017 [hep-ph/0604192] [INSPIRE].

[39] M. Asano and T. Higaki, Natural supersymmetric spectrum in mirage mediation, Phys. Rev. D 86 (2012) 035020 [arXiv:1204.0508] [INSPIRE]. 
[40] X. Kang Du, G.-L. Liu, F. Wang, W. Wang, J.M. Yang and Y. Zhang, NMSSM from generalized deflected mirage mediation, arXiv:1804.07335 [INSPIRE].

[41] H. Baer, V. Barger, P. Huang, A. Mustafayev and X. Tata, Radiative natural SUSY with a 125 GeV Higgs boson, Phys. Rev. Lett. 109 (2012) 161802 [arXiv:1207.3343] [INSPIRE].

[42] A. Mustafayev and X. Tata, Supersymmetry, Naturalness and Light Higgsinos, Indian J. Phys. 88 (2014) 991 [arXiv:1404.1386] [INSPIRE].

[43] K.J. Bae, H. Baer, N. Nagata and H. Serce, Prospects for Higgs coupling measurements in SUSY with radiatively-driven naturalness, Phys. Rev. D 92 (2015) 035006 [arXiv: 1505. 03541] [INSPIRE].

[44] X. Tata, Supersymmetry: Aspirations and Prospects, Phys. Scripta 90 (2015) 108001 [arXiv: 1506.07151] [INSPIRE].

[45] R. Kitano, G.D. Kribs and H. Murayama, Electroweak symmetry breaking via UV insensitive anomaly mediation, Phys. Rev. D 70 (2004) 035001 [hep-ph/0402215] [INSPIRE].

[46] X. Ning and F. Wang, Solving the muon $g-2$ anomaly within the NMSSM from generalized deflected AMSB, JHEP 08 (2017) 089 [arXiv:1704.05079] [INSPIRE].

[47] K. Choi, K.S. Jeong, S. Nakamura, K.-I. Okumura and M. Yamaguchi, Sparticle masses in deflected mirage mediation, JHEP 04 (2009) 107 [arXiv:0901.0052] [INSPIRE].

[48] B. Altunkaynak, B.D. Nelson, L.L. Everett, I.-W. Kim and Y. Rao, Phenomenological Implications of Deflected Mirage Mediation: Comparison with Mirage Mediation, JHEP 05 (2010) 054 [arXiv: 1001.5261] [INSPIRE].

[49] G.F. Giudice and R. Rattazzi, Extracting supersymmetry breaking effects from wave function renormalization, Nucl. Phys. B 511 (1998) 25 [hep-ph/9706540] [INSPIRE].

[50] Z. Chacko and E. Ponton, Yukawa deflected gauge mediation, Phys. Rev. D 66 (2002) 095004 [hep-ph/0112190] [INSPIRE].

[51] J.A. Evans and D. Shih, Surveying Extended GMSB Models with $m_{h}=125 \mathrm{GeV}$, JHEP 08 (2013) 093 [arXiv: 1303.0228] [INSPIRE].

[52] M. Dine, W. Fischler and M. Srednicki, Supersymmetric Technicolor, Nucl. Phys. B 189 (1981) 575 [INSPIRE].

[53] S. Dimopoulos and S. Raby, Supercolor, Nucl. Phys. B 192 (1981) 353 [inSPIRE].

[54] M. Dine and W. Fischler, A Phenomenological Model of Particle Physics Based on Supersymmetry, Phys. Lett. B 110 (1982) 227 [INSPIRE].

[55] M. Dine and A.E. Nelson, Dynamical supersymmetry breaking at low-energies, Phys. Rev. D 48 (1993) 1277 [hep-ph/9303230] [INSPIRE].

[56] M. Dine, A.E. Nelson and Y. Shirman, Low-energy dynamical supersymmetry breaking simplified, Phys. Rev. D 51 (1995) 1362 [hep-ph/9408384] [INSPIRE].

[57] M. Dine, A.E. Nelson, Y. Nir and Y. Shirman, New tools for low-energy dynamical supersymmetry breaking, Phys. Rev. D 53 (1996) 2658 [hep-ph/9507378] [INSPIRE].

[58] G.F. Giudice and R. Rattazzi, Theories with gauge mediated supersymmetry breaking, Phys. Rept. 322 (1999) 419 [hep-ph/9801271] [INSPIRE].

[59] A.E. Nelson and N.J. Weiner, Extended anomaly mediation and new physics at 10-TeV, hep-ph/0210288 [inSPIRE]. 
[60] X. Du and F. Wang, NMSSM From Alternative Deflection in Generalized Deflected Anomaly Mediated SUSY Breaking, Eur. Phys. J. C 78 (2018) 431 [arXiv:1710.06105] [INSPIRE].

[61] F. Wang, Deflected anomaly mediated SUSY breaking scenario with general messenger-matter interactions, Phys. Lett. B 751 (2015) 402 [arXiv:1508.01299] [INSPIRE].

[62] X. Ning and F. Wang, Solving the muon $g-2$ anomaly within the NMSSM from generalized deflected AMSB, JHEP 08 (2017) 089 [arXiv:1704.05079] [INSPIRE].

[63] F. Wang, W. Wang and J.M. Yang, Solving the muon $g-2$ anomaly in deflected anomaly mediated SUSY breaking with messenger-matter interactions, Phys. Rev. D 96 (2017) 075025 [arXiv: 1703.10894] [INSPIRE].

[64] B. Kaufman and B.D. Nelson, Mirage Models Confront the LHC: II. Flux-Stabilized Type IIB String Theory, Phys. Rev. D 89 (2014) 085029 [arXiv:1312.6621] [InSPIRE]. 